\title{
Kaotik Harita Temelli Ağaç Tohum Algoritması
}

\author{
Burhanettin DURMUŞ*1
}

${ }^{1}$ Kütahya Dumlupınar Üniversitesi, Mühendislik Fakültesi, Elektrik-Elektronik Bölümü, 43100, Kütahya, Türkiye

(Alınış / Received: 24.04.2019, Kabul / Accepted: 01.08.2019, Online Yayınlanma / Published Online: 30.08.2019)

Anahtar Kelimeler

Ağaç tohum algoritması,

Kaotik haritalar,

Sezgisel algoritmalar
Özet: Kaotik haritalama tekniklerinin sezgisel algoritmalarda rastgele sayı üreteci olarak kullanımı giderek yaygınlaşan bir konudur. Geniş bir spekturuma sahip bu haritalama teknikler, sezgisel algoritmaların rastgele seçimlerindeki çeşitliliği arttırarak performans artışı sağlamaktadırlar. Ağaç tohum algoritması (TSA), son dönemde önerilmiș popülasyon temelli sezgisel algoritmalardan biridir. Doğadaki ağaç ve tohum gelişimini ilham alan bu algoritma, hesapsal süreci boyunca rastgele sayı dizilerini kullanan işlem basamaklarına sahiptir. Bu çalışmada, kaotik haritalama kullanılarak TSA 'nın performansında iyileștirmeye odaklanılmıștır. Beș farklı kaotik harita temelli TSA (CTSA) metodu geliştirilmiștir. Geliştirilen metotların performansları 24 adet test fonksiyonu üzerinden karşılaştırılmıştır. Elde edilen sonuçlar, kaotik haritalamanın TSA'nın yakınsama ve lokal optimumdan kaçış performansına katkı sağladığını göstermektedir.

\section{Chaotic Map Based Tree Seed Algorithm}

\section{Keywords}

Tree seed algorithm,

Chaotic maps,

Metaheuristics

\begin{abstract}
The use of chaotic maps as a random number generator in metaheuristics is a common issue. These methods, which have a spread spectrum, increase the diversity in the random selection of heuristic algorithms, resulting in increased performance. Tree seed algorithm (TSA) is one of the recently proposed population-based metaheuristic algorithms. Inspired by the growth of trees and seeds in nature, this algorithm has processing phases that use random numbers throughout the computational process. This paper focuses on improving the performance of the TSA using chaotic mapping. Five chaotic based TSA's (CTSA's) are developed. The developed methods are benchmarked on 24 test functions. The obtained results show that chaotic mapping contributes to the performance of TSA in terms of both local optima avoidance and convergence speed.
\end{abstract}

\section{Giriș}

Son yirmi yldır optimizasyon problemlerinin çözümünde sezgisel algoritmalar kayda değer sonuçlar üretmektedirler. Doğadaki canlıların yaşamsal veya karar verme gibi davranış biçimlerinden ilham alınarak geliştirilen bu metotlarda, en iyi sonuca ulaşma hedefiyle hesapsal süreç iterasyonlar boyunca sürdürülür. Bu hesapsal süreçte yeni çözüm üretimi veya gelişimi farklı stratejilere bağlıdır. Parçacık sürü optimizasyonu (PSO) kuş ve balık sürülerinin sosyal ve bireysel hareketleri gözönüne alınarak geliştirilmiştir [1]. Karınca koloni optimizasyonunda (ACO), karıncaların salgıladığı feremon ile en kısa yolu izleme dürtüleri taklit edilmiştir [2]. Yapay arı kolonisi (ABC), arıların yiyecek kaynağı arayışındaki zeki davranışları taklit edilerek geliştirilmiştir [3]. Çiçek tozlaşma algoritmasında (FPA), çiçeklerin biyolojik tozlaşma süreci model alınmıştır [4]. Aslan optimizasyon algoritması (LOA), aslanların avlanırken sergiledikleri işbirliğini ilham alır [5]. Yapısal farlılıkları olmasına rağmen tüm bu sezgisellerde hesapsal sürecin bazı aşamalarında rasgele seçim işlemlerine ihtiyaç duyulmaktadır. Bu rastgele seçim işlemlerinde genel olarak uniform dağılıma sahip rastgele sayı dizileri kullanılmaktadır. Böyle bir dağılım, üretilen rastgele sayıların belli alanlarda toplanmasına yol açabilmektedir. Bu durum rastgele sayı dizilerini kullanan sezgisel algoritmaların performansı açısından değerlendirildiğinde ise, algoritmanın yeni birey üretimindeki çeşitliliğin azalmasına veya yerel minimumlara takılmasına sebebiyet verebilir. Daha yaygin spekturuma sahip rastgele sayı dizilerinin kullanımı söz konusu olumsuzlukların giderilmesine yardımcı olacaktır. 
Kaotik haritalamanın sezgisel algoritmalarda rastgele sayı üreteci olarak kullanımına olan ilgi giderek artmaktadır. Kaotik denklemlerin ateş böceği algoritmasına uyarlandığı bir çalışmada (FA), algoritmanın performansının arttırdığı gösterilmiştir. [6]. Yerçekimsel arama algoritmasının (GSA) kaotik denklem kullanılarak modifiye edildiği bir diğer çalışmada, filtre katsayı kestiriminin daha doğru yapıldığı vurgulanmıştır [7]. Diğer bir çalışmada, karides sürüsü algoritmasının $(\mathrm{KH})$ temel ayar parametrelerinin kaotik haritalar ile belirlendiği ve algoritmanın yakınsama davranışını iyileştirdiği gösterilmiştir [8]. Armoni arama algoritması (HS) farklı kaotik haritalama teknikleri ile modifiye edilmiştir [9]. Rastgele sayıların bu haritalar ile üretimi HS algoritmasının daha hızlı yakınsamasını sağlamıştır. Bir diğer çalışmada, elektromekanik modele ait parametre kestirimi için kaotik haritalı geri izleme algoritması (BSA) geliştirilmiştir [10]. Elde edilen sonuçlar geliştirilen metodun parametre kestiriminde daha başarılı olduğunu göstermektedir. Balina optimizasyon algoritmasının (WOA) geliştirildiği diğer bir çalışmada, algoritma parametreleri farklı kaotik haritalar ile belirlenmektedir. Geliştirilen algoritmanın test fonksiyonlarında daha iyi sonuçlar verdiği rapor edilmiştir [11]. Kaotik haritaların gri kurt optimizasyon algoritmasına (GWO) modifiye edildiği diğer bir çalışmada, önerilen metodun kısıtlı optimizasyon problemlerinin çözümünde iyi sonuçlar ürettiği ifade edilmiştir [12]. Kaotik temelli diferansiyel gelişim algoritması (DE), kaotik optimizasyon algoritması (COA), kaotik haritalı biyocoğrafya temelli optimizasyon (BBO) gibi çalışmalar, kaotik sistemlerin algoritma gelişiminde kullanıldığı çalışmalara örnek gösterilebilir [13-15]. Sonuç olarak kaotik haritalama, geliştirilen algoritmaların yakınsama ve lokal minumumdan kaçma kabiliyetlerini arttırmaktadır.

Ağaç tohum algoritması (TSA), son dönemde önerilen sezgisellerden biri olup ağaçların gelişimi ve tohumlarının üretilmesi sürecinden ilham alınarak geliştirilmiştir [16]. Bu algoritmada ağaç ve tohum konumlarının bir arama uzayında optimum çözüme ulaşması amaçlanır. Uygunluğu daha iyi konuma sahip tohumların ağaçlar ile yer değiştirmesi yolu ile gelişim iterasyonlar boyunca sürdürülür. TSA ilk olarak sürekli zaman optimizasyon problemlerine uygulanmıştır [16]. Önerilen çalışmada, 5 boyutlu test fonksiyonları üzerinden TSA ve diğer sezgisel algoritmalar ile karşılaştırmalar sunulmuştur. Ayrıca literatürde TSA ile ilgili sadece birkaç çalışmaya rastlanmıştır [17-19]. Bundan dolayı bu çalışmada TSA'nın performansını arttırmaya odaklanılmıştır. Literatürde, TSA geliştirmeye yönelik çalışmalar şu şekilde özetlenebilir; TSA'nın grafik işlem birimi üzerinde paralel versiyonu geliștirilmiş [17]. Paralel versiyonun kıyas fonksiyonları üzerinden seri versiyonuna göre performansı incelenmiş ve problemin türüne bağlı olarak daha iyi sonuçların üretilebileceği gösterilmiştir. Yazarın yer aldığı diğer bir çalışmada da [18] kısıtlı optimizasyon için TSA modifikasyonu geliştirilmiştir. Geliştirilen metot mühendislik problemlerine uygulanmıștır. Bir diğer çalışmada da TSA'nın binary versiyonu geliştirilmiştir. Hibrit yaklaşımların TSA'nın performansında artış sağlayacağı vurgulanmıștır [19].

Bu çalışmada, kaotik temelli haritalama metotları TSA algoritmasına uyarlanmıştır. Literatürde kabul görmüş beş adet kaotik haritalama ile kaotik temelli TSA (CTSA) metotları türetilmiștir. TSA'nın hesapsal sürecinde ihtiyaç duyulan rastgele seçim işlemlerinde bu kaotik haritalamalardan elde edilen sayı dizileri kullanılmıştır. Türetilen CTSA metotların ve klasik TSA'nın performansı test fonksiyonları üzerinden değerlendirilmiş, elde edilen nümeriksel sonuçlar ve yakınsama eğrileri karşılaştırılmıştır.

\section{TSA Algoritması}

TSA algoritması doğadan esinlenerek geliştirilen sezgisel algoritmalardan biridir. Bu algoritmada, ağaç ve tohumların belli bir alan içerisindeki konumları optimizasyon problemi olarak değerlendirilmektedir [16]. Her bir tohum üretimi ağaç popülasyonundaki en iyi veya rastgele seçilen bir ağaç konumuna bağlı olarak gerçekleştirilir. Hesapsal süreç ağaçların başlangıç konumlarının aşağıdaki denklem kullanılarak belirlenmesi ile başlatılır.

$$
T_{i, j}=L_{j, \min }+\operatorname{rand}(0,1)\left(H_{j, \max }-L_{j, \min }\right)
$$

Burada, $i=1,2 \ldots N$, ve $j=1,2, \ldots D$ olmak üzere; $T_{i, j} j$ boyutlu $i$. ağacın konumunu, $H_{j, \max }$ ve $L_{j, \max }$ arama uzayı üst ve alt sınırını, $N$ ağaç sayısını, $D$ boyutu, rand ise rastgele sayı değerini temsil eder.

Ağaç konumları üretildikten sonra her bir konum optimizasyon probleminin amaç fonksiyonuna göre değerlendirilir. Her bir ağaç için tohum üretimi iki farklı şekilde gerçekleştirilir. Bu seçim arama eğilimi (ST) olarak tanımlanan algoritmanın en temel kontrol parametresi üzerinden belirlenir. Üretilen rastgele değer bu parametreden daha düşük ise Denklem 2, daha büyük ise Denklem 3'deki tohum üretim mekanizması kullanılır.

$$
\begin{gathered}
S_{i, j}=T_{i, j}+\operatorname{rand}(-1,1) \times\left(B_{j}-T_{r, j}\right) \\
S_{i, j}=T_{i, j}+\operatorname{rand}(-1,1) \times\left(T_{i, j}-T_{r, j}\right)
\end{gathered}
$$

Burada, $S i$. tohumun $j$. boyutunu, $T i$. ağacın $j$. boyutunu, $B_{j}$ en iyi ağacın $j$. boyutunu, $T_{r j}$ popülasyondan rastgele seçilmiş $i$. ağacın $j$. boyutunu temsil eder.

Üretilen tohumlar amaç fonksiyonuna göre değerlendirildikten sonra mevcut ağaç konumundan daha iyi uygunluğa sahip olanları sonraki nesilde ağaçların yerini alırlar. Tohum üretimi ve gelişim 
süreci maksimum fonksiyon çağırma sayısına (FES) ulaşllıncaya kadar sürdürülür [16].

TSA genel olarak değerlendirildiğinde başarılı sonuçlar üretmesine rağmen, rastgele seçimlerin daha etkin olduğu bir hesapsal sürece sahip olduğu görülmektedir [16]. Bu durumda, birbirine benzeyen rastgele sayıların kullanımı genetik çeşitliliğin sürdürülmesini engelleyebilmektedir. Bunun sonucunda da üretilen çözümlerin lokal minimuma takılmaları kaçınılmaz olmaktadır. Öte yandan, TSA'da yeni birey üretimi tohum bireyler üzerinden gerçekleşmekte, ağaç sayısı düşük tutulmaktadır. Diğer bir deyişle popülasyon sayısı için küçük değerler seçilmektedir [16]. Aynı çalışmadaki sonuçlar değerlendirildiğinde, popülasyon sayısının arttırılması ile performansın düştügü gösterilmiștir [16]. Bunun nedeni, artan popülasyon sayısının maximum fonksiyon çağrı sayısı (maxFES) bütçesini hızlıca tüketmesidir. Bunun sonucunda algoritmanın yakınsama ve lokal arama kabiliyetleri zayıflayabilmektedir. Bahsedilen olumsuzlukları giderebilmek, TSA'nın yakınsama ve lokal arama yönlerini güçlendirmek için bu çalışmada kaotik temelli TSA geliştirmeye odaklanılmıştır.

\section{Kaotik Temelli TSA}

Klasik TSA algoritmasında, diğer sezgisel algoritmalar gibi rastgele konumlandırılmış bireylerden oluşan başlangıç havuzunun üretilmesi ile hesapsal sürece başlanır. Daha sonra Denklem 2 ve 3'de ifade edilen tohum üretimi işlemi gerçekleştirilir. Rastgele seçimlerin yapıldığı bu işlemlerde, yeni iterasyona aday olacak ağaç konumları belirlenerek gelişim sürdürülür. Rasgele seçimlerde, uniform dağılıma sahip rastgele üretilen sayı dizileri kullanılmaktadır. Bu çalışmada, TSA'nın tohum üretimindeki çeşitliliği arttırmaya ve yerel minimumdan kaçma kabiliyetini güçlendirmeye odaklanılmıștır. TSA'nın hesapsal sürecinde ihtiyaç duyulan rastgele sayı üretimi kaotik haritalama metotları kullanılarak üretilmektedir. Literatürde yer alan farklı kaotik haritalama metotları TSA algoritması ile birleştirilmiş ve 5 adet kaotik temelli TSA (CTSA) algoritması türetilmiștir. Seçilen haritalama metotları aşağıdaki alt başlıklarda sunulmuştur.

\subsection{Gauss/Mouse haritalı TSA (CTSA-1)}

Gauss/Mouse haritalama, Gauss fonksiyonu ile elde edilen bir aralıktaki gerçel sayıların doğrusal olmayan dağılımını üretir [20]. Denklem 4 'de haritanın kaotik denklemi, Şekil 1 'de ise haritanın davranışı verilmiştir.

$$
x_{i+1}=\left\{\begin{array}{cc}
1 & x_{i+1} \\
\frac{1}{\bmod \left(x_{i}, 1\right)} & \text { aksihalde }
\end{array}\right.
$$

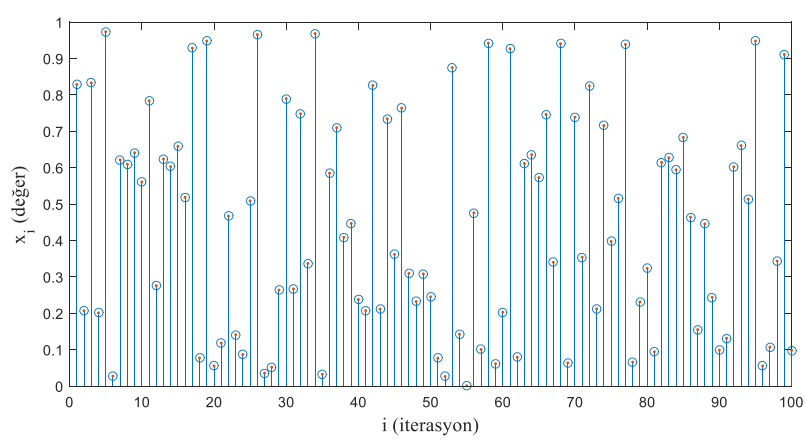

Şekil 1. Gauss/Mouse haritalama

\subsection{Intermittency haritalı TSA (CTSA-2)}

$\mathrm{Bu}$ haritalama, çok sayıdaki iteratifsel unsur içeren durumlarda alt ve üst sınırları aşmayan davranış sergileyen bir haritadır. Biri doğrusal diğeri doğrusal olmayan iki kısma sahip olup, kaotik denklemi ve davranıș değişim aşağıda verilmiştir [21].

$$
x_{i+1}=\left\{\begin{array}{cl}
\varepsilon+x_{i}+c x_{i}^{n} & 0<x_{i}<p, \\
\frac{x_{i}-p}{1-p} & p<x_{i}<1 .
\end{array}\right.
$$

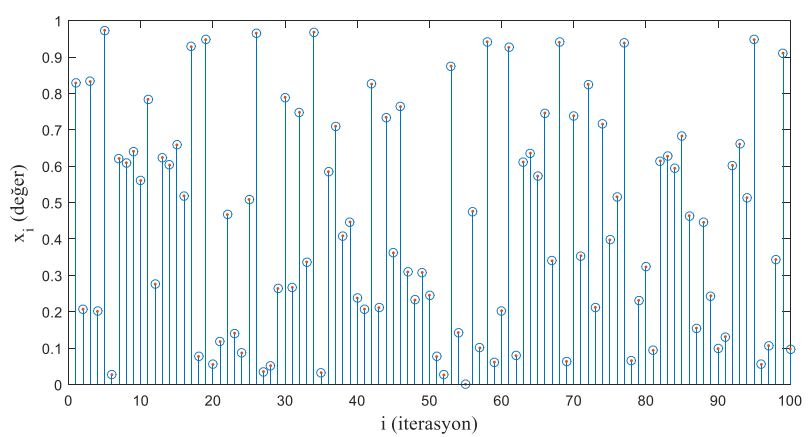

Şekil 2. Intermittency haritalama

\subsection{Sinüzoidal haritalı TSA (CTSA-3)}

Sinüzoidal haritalama kaotik sayı üretiminde yaygın bir kullanıma sahip olup denklem ve değișimi așağıda [22] gösterilmiştir.

$$
x_{i+1}=a x_{i}^{2} \sin \left(\pi x_{i}\right)
$$

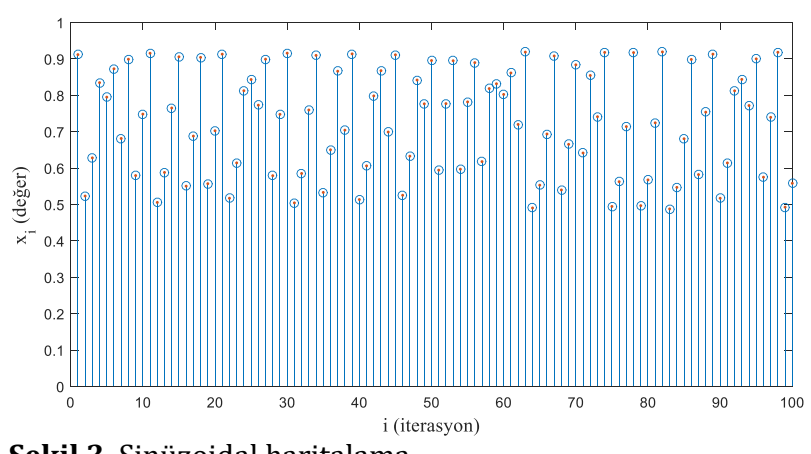

Şekil 3. Sinüzoidal haritalama 


\subsection{Standart haritalı TSA (CTSA-4)}

Standart haritalama, iki adet kanonik değişkene bağlı olarak değer üreten bir haritalamadır ve Denklem 7‘de verilen kaotik denklemi kullanır [23].

$$
\begin{gathered}
x_{i+1}=x_{i}+K \sin \left(y_{i}\right) \\
y_{i+1}=y_{i}+x_{i+1}
\end{gathered}
$$

Burada $K$ kaos derecesini etkileyen boyutsuz bir parametredir. Sinüs fonksiyonunun periyodikliği nedeniyle değișkenler bir silindirik yörüngede düşünülebilir. Standart haritanın davranış değişimi Şekil 4'de gösterilmiştir.

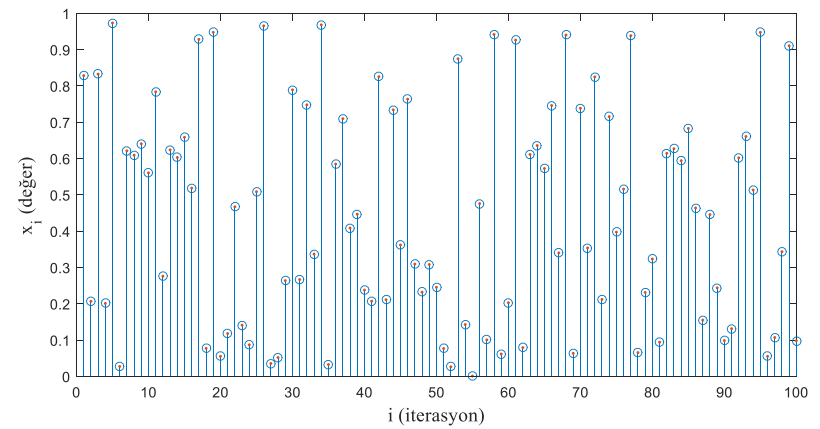

Şekil 4. Standart haritalama

\subsection{Zaslavskii haritalı TSA (CTSA-5)}

$\mathrm{Bu}$ haritalama Lyapunov üs alma ve geniş-spektrum davranışına sahip bir dağılıma sahiptir. Değişkenlerden birine göre durum alanının periyodikliği ile karakterize edilen bir dağılım dinamiğini belirler [24]. Haritanın kaotik denklemi ve davranış değişimi aşağıda gösterilmiştir.

$$
\begin{gathered}
x_{i+1}=\left[x_{i}+v\left(1+\mu y_{i}\right)\right. \\
\left.+\varepsilon v \mu \cos \left(2 \pi x_{i}\right)\right] \bmod (1) \\
y_{i+1}=e^{-\gamma}\left(y_{i}+\varepsilon \cos \left(2 \pi x_{i}\right)\right)
\end{gathered}
$$

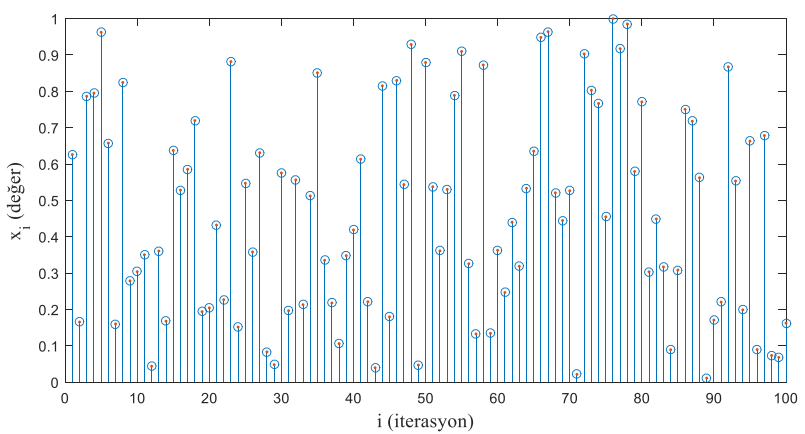

Şekil 5. Zaslavskii haritalama

Yukarıdaki alt başlıklarda anlatılan kaotik haritalama metotları rastgele sayı üretiminde kullanılmaktadır. Beş farklı haritalama metodundan seçilen bir kaotik denklemin ürettiği rastgele sayı değerleri $\left(x_{i+1}\right)$, TSA'nın hesapsal sürecinde kullanılmaktadır. Bașlangıç, seçim ve tohum üretimi aşamalarında ihtiyaç duyulan rastgele değerler için bu haritalardan elde edilen kaotik değerler kullanılmaktadır. Böylece kendini tekrarlayan uniform sayılar yerine farklı kaotik rastgele sayılar kullanılmaktadır.

\section{Simülasyon Sonuçları}

Sezgisel algoritmaların performans karşılaştırmaları genel olarak farklı karakteristiklere sahip test fonksiyonları üzerinden yapılır. $\mathrm{Bu}$ bağlamda, önerilen CTSA metotlarının performansını izlemek için iki aşamalı simülasyon çalışması yürütülmüştür. İlk olarak, literatürde sunulan orijinal TSA algoritması için kullanılan 5-boyutlu 24 adet test fonksiyonu üzerinden karşılaștırmalar yapılmıștır. Test fonksiyonlarının matematiksel tanımları ve özellikleri ilgili makalede ve diğer makalelerde yer almaktadır [16, 25-27].

Doğru bir karşılaştırma için TSA parametreleri; birey sayısı $P o p=10, S T=0.1$ ve maxFES $=50000$ olarak belirlenmiștir [16]. Algoritmaların 30 bağımsız denemeden elde edilen ortalama ve standart sapma (S. S.) sonuçları Tablo 1 'de sunulmuştur. İlaveten DE ve HS algoritmalarına ait sonuçlarda aynı tabloda verilmiştir. İkinci olarak ise önerilen metotların yakınsama davranışlarını incelemek için problem boyutu $(D) 10$ ve 100 olarak belirlenmiștir. Bu durumda, $\max F E S=D \times 10000$ olarak değiștirilmiștir. 30 bağımsız deneme sonucu elde edilen en iyi, ortalama ve standart sapma sonuçları 10-boyutlu problemler için Tablo 2'de, 100-boyutlu problemler için Tablo 3'de verilmiştir. Ayrıca yakınsama davranışlarını incelemek üzere, türetilen algoritmaların yakınsama eğrileri 10-boyutlu problemler için Şekil 6 ve 7'de gösterilmiștir.

Tablo 1'de verilen sonuçların ışı̆̆ında türetilen CTSA metotları genel olarak klasik TSA'ya göre daha düşük hata seviyelerine ulaşmışlardır. Özellikle $F_{12}, F_{14}, F_{15}$, $F_{17}$ ve $F_{19}$ gibi multimodal fonksiyonlarda CTSA algoritmaları ile elde edilen performans artışı daha açlktır. $F_{4}, F_{8}, F_{11}$ ve $F_{20}$ fonksiyonlar için tüm algoritmalar global minimuma ulaşmışlardır.

Tablo 2'deki veriler incelendiğinde unimodal fonksiyonlarda genel olarak sonuçların birbirine çok yakın olduğu görülmektedir. Multimodal fonksiyonlarda ise lokal minimumlara takılmalar olmasına rağmen $F_{11}, F_{13}$ ve $F_{23}$ gibi fonksiyonlarda kaotik temelli algoritmalar daha düşük hata seviyelerini ulaşmayı başarmışlardır.

Tablo 3'deki veriler incelendiğinde, unimodal fonksiyonlarda benzer sonuçlar elde edilmiștir. Multimodal fonksiyonlar için ise algoritmaların lokal optimumlara takıldıkları görülmektedir. Artan problem boyutu beraberinde lokal optimum sayısını da arttırmıştır. Genel olarak değerlendirildiğinde, türetilen CTSA algoritmaları bu tür problemlerde daha iyi sonuçlar üretmektedir.

Şekil 6 ve 7'deki yakınsama eğrileri incelendiğinde önerilen CTSA metotları daha hızlı yakınsama davranışı göstermişlerdir. Unimodal fonksiyonlarda 
yakınsama kabiliyetinin yüksek olması ve algoritmanın global optimum komşuluğuna hızlı bir şekilde ulaşması istenir [28]. Bu bağlamda unimodal fonksiyonlar için CTSA-4 diğer metotlara göre çok daha hızlı bir şekilde yakınsama karakteristiği göstermektedir. Yakınsama performansında CTSA-4 metodunu CTSA-1 ve CTSA-3 metotları izlemektedir. Multimodal fonksiyonlarda ise algoritmanın lokal minimumlardan kaçma kabiliyetini yansıttı̆̆ için sonuç değerleri daha önemlidir. Son değerler incelendiğinde CTSA-2, CTSA-4 ve CTSA-5 metotları diğer metotlara göre daha düşük seviyede hata değerleri üretmişlerdir.

\section{Tartışma ve Sonuç}

$\mathrm{Bu}$ çalışmada, farklı karakteristiklere sahip test fonksiyonları kullanılarak yapılan simülasyon çalışmalarından elde edilen sonuçlar ile kaotik denklemleri kullanan rastgele sayı dizilerinin sezgisel algoritmaların performansında artış sağladığ gösterilmektedir. Kaotik harita temelinde geliştirilen metotlar, TSA algoritmasının yeni birey üretimindeki çeşitliliğin arttırılmasına ve lokal optimumlardan kaçmasına yardımcı olmaktadır. Unimodal fonksiyonlarda hızlı bir yakınsama, multimodal fonksiyonlarda ise daha düşük hata seviyelerinin elde edilmesi bu sonucu desteklemektedir. Haritalama çeşitleri arasında karşılaştırma yapıldı̆̆ında ise standart haritalamanın daha uygun olduğu görülmüştür. Sonraki çalışmalarda, kısıtlı optimizasyon ve gerçek dünya problemlerin çözümünde kaotik haritalamanın etkilerinin incelenmesi hedeflenmektedir.

Tablo 1. 5-boyutlu test fonksiyonları için elde edilen sonuçlar

\begin{tabular}{|c|c|c|c|c|c|c|c|c|c|}
\hline$F$ & İndeks & TSA [12] & CTSA-1 & CTSA-2 & CTSA-3 & CTSA-4 & CTSA-5 & $\mathrm{DE}$ & HS \\
\hline \multirow{2}{*}{$F_{1}$} & Ortalama & $7.64 \mathrm{E}-243$ & $2.83 \mathrm{E}-250$ & $2.84 \mathrm{E}-250$ & $1.92 \mathrm{E}-249$ & $1.64 \mathrm{E}-252$ & $9.83 E-246$ & $2.78 \mathrm{E}+001$ & 8.94E-006 \\
\hline & S. S. & $0.00 \mathrm{E}+00$ & $0.00 \mathrm{E}+00$ & $0.00 E+00$ & $0.00 \mathrm{E}+00$ & $0.00 \mathrm{E}+00$ & $0.00 \mathrm{E}+00$ & $3.64 \mathrm{E}+001$ & 7.95E-006 \\
\hline \multirow[b]{2}{*}{$F_{2}$} & Ortalama & $3.85 \mathrm{E}-240$ & $2.82 \mathrm{E}-246$ & $5.63 \mathrm{E}-246$ & $1.41 \mathrm{E}-244$ & $2.74 \mathrm{E}-246$ & $3.82 E-247$ & $7.10 \mathrm{E}+003$ & $2.69 \mathrm{E}+000$ \\
\hline & S. S. & $0.00 \mathrm{E}+00$ & $0.00 \mathrm{E}+00$ & $0.00 E+00$ & $0.00 \mathrm{E}+00$ & $0.00 \mathrm{E}+00$ & $0.00 E+00$ & $1.25 \mathrm{E}+002$ & $2.82 \mathrm{E}+000$ \\
\hline \multirow{2}{*}{$F_{3}$} & Ortalama & $5.44 \mathrm{E}-239$ & $4.68 E-252$ & 1.33E-249 & $1.42 \mathrm{E}-250$ & 1.18E-249 & 4.57E-249 & $4.20 \mathrm{E}+000$ & $2.32 \mathrm{E}-007$ \\
\hline & S.S & $0.00 \mathrm{E}+00$ & $0.00 \mathrm{E}+00$ & $0.00 E+00$ & $0.00 \mathrm{E}+00$ & $0.00 \mathrm{E}+00$ & $0.00 \mathrm{E}+00$ & $6.36 \mathrm{E}+00$ & $1.43 \mathrm{E}-07$ \\
\hline \multirow{2}{*}{$F_{4}$} & Ortalama & $0.00 \mathrm{E}+00$ & $0.00 \mathrm{E}+00$ & $0.00 E+00$ & $0.00 \mathrm{E}+00$ & $0.00 \mathrm{E}+00$ & $0.00 \mathrm{E}+00$ & $2.71 \mathrm{E}-07$ & 4.96E-09 \\
\hline & S. S. & $0.00 \mathrm{E}+00$ & $0.00 E+00$ & $0.00 E+00$ & $0.00 \mathrm{E}+00$ & $0.00 E+00$ & $0.00 \mathrm{E}+00$ & $1.40 \mathrm{E}-07$ & 6.96E-09 \\
\hline \multirow{2}{*}{$F_{5}$} & Ortalama & $3.49 \mathrm{E}-147$ & $2.94 \mathrm{E}-150$ & $2.21 \mathrm{E}-149$ & $9.01 \mathrm{E}-151$ & $6.41 \mathrm{E}-150$ & 7.58E-151 & $2.32 \mathrm{E}-01$ & $6.54 \mathrm{E}-04$ \\
\hline & S. S. & $1.75 \mathrm{E}-146$ & $4.49 \mathrm{E}-150$ & 2.97E-149 & $2.12 \mathrm{E}-150$ & $1.50 \mathrm{E}-149$ & 1.15E-150 & $3.60 \mathrm{E}-01$ & $1.43 \mathrm{E}-04$ \\
\hline \multirow{2}{*}{$F_{6}$} & Ortalama & $9.47 \mathrm{E}-63$ & 4.58E-69 & $3.04 \mathrm{E}-68$ & $1.75 \mathrm{E}-68$ & $2.03 \mathrm{E}-68$ & 8.36E-69 & $3.52 \mathrm{E}-01$ & $5.18 \mathrm{E}-03$ \\
\hline & S. S. & $5.15 \mathrm{E}-62$ & $6.64 E-69$ & $9.02 E-68$ & $4.78 \mathrm{E}-68$ & $5.88 \mathrm{E}-68$ & $2.22 \mathrm{E}-68$ & $6.83 \mathrm{E}-01$ & $1.56 \mathrm{E}-03$ \\
\hline \multirow{2}{*}{$F_{7}$} & Ortalama & 3.33E-02 & $0.00 \mathrm{E}+00$ & $0.00 E+00$ & $0.00 \mathrm{E}+00$ & $0.00 \mathrm{E}+00$ & $0.00 E+00$ & $1.05 \mathrm{E}+01$ & $0.00 \mathrm{E}+00$ \\
\hline & S. S. & $1.83 \mathrm{E}-01$ & $0.00 \mathrm{E}+00$ & $0.00 \mathrm{E}+00$ & $0.00 \mathrm{E}+00$ & $0.00 \mathrm{E}+00$ & $0.00 \mathrm{E}+00$ & $6.89 \mathrm{E}+00$ & $0.00 \mathrm{E}+00$ \\
\hline \multirow{2}{*}{$F_{8}$} & Ortalama & $0.00 \mathrm{E}+00$ & $0.00 \mathrm{E}+00$ & $0.00 E+00$ & $0.00 \mathrm{E}+00$ & $0.00 \mathrm{E}+00$ & $0.00 \mathrm{E}+00$ & 4.70E-03 & $1.69 \mathrm{E}-18$ \\
\hline & S. S. & $0.00 \mathrm{E}+00$ & $0.00 \mathrm{E}+00$ & $0.00 \mathrm{E}+00$ & $0.00 \mathrm{E}+00$ & $0.00 \mathrm{E}+00$ & $0.00 \mathrm{E}+00$ & 7.49E-03 & $2.82 \mathrm{E}-18$ \\
\hline \multirow{2}{*}{$F_{9}$} & Ortalama & $5.16 \mathrm{E}-04$ & $3.57 \mathrm{E}-04$ & $4.38 \mathrm{E}-04$ & 5.39E-04 & $2.86 \mathrm{E}-04$ & $3.88 \mathrm{E}-04$ & $3.32 \mathrm{E}-03$ & $1.01 \mathrm{E}-03$ \\
\hline & S. S. & $3.05 \mathrm{E}-04$ & $3.15 \mathrm{E}-04$ & $1.39 \mathrm{E}-04$ & 1.33E-04 & $1.64 \mathrm{E}-04$ & $1.69 \mathrm{E}-04$ & $1.02 \mathrm{E}-03$ & $6.16 \mathrm{E}-04$ \\
\hline \multirow{2}{*}{$F_{10}$} & Ortalama & $3.15 \mathrm{E}-01$ & $1.08 \mathrm{E}-01$ & $3.52 \mathrm{E}-01$ & $6.69 \mathrm{E}-01$ & 8.89E-02 & 5.83E-01 & $1.59 \mathrm{E}+02$ & $1.69 \mathrm{E}+00$ \\
\hline & S. S. & $1.05 \mathrm{E}+00$ & $6.67 \mathrm{E}-02$ & 5.93E-01 & $5.24 \mathrm{E}-01$ & 3.81E-02 & $1.08 \mathrm{E}+00$ & $2.49 \mathrm{E}+02$ & $1.20 \mathrm{E}+00$ \\
\hline \multirow{2}{*}{$F_{11}$} & Ortalama & $0.00 \mathrm{E}+00$ & $0.00 \mathrm{E}+00$ & $0.00 \mathrm{E}+00$ & $0.00 \mathrm{E}+00$ & $0.00 \mathrm{E}+00$ & $0.00 \mathrm{E}+00$ & $3.98 \mathrm{E}+00$ & $5.01 \mathrm{E}-06$ \\
\hline & S. S. & $0.00 \mathrm{E}+00$ & $0.00 E+00$ & $0.00 E+00$ & $0.00 \mathrm{E}+00$ & $0.00 \mathrm{E}+00$ & $0.00 \mathrm{E}+00$ & $1.14 \mathrm{E}+00$ & $3.88 \mathrm{E}-06$ \\
\hline \multirow[b]{2}{*}{$F_{12}$} & Ortalama & 4.67E-01 & 3.00E-01 & $0.00 E+00$ & $0.00 \mathrm{E}+00$ & $0.00 \mathrm{E}+00$ & $4.00 \mathrm{E}-01$ & $3.53 \mathrm{E}+00$ & $4.78 \mathrm{E}-06$ \\
\hline & S. S. & $5.71 \mathrm{E}-01$ & $4.58 \mathrm{E}-01$ & $0.00 E+00$ & $0.00 \mathrm{E}+00$ & $0.00 E+00$ & 4.89E-01 & $1.34 \mathrm{E}+00$ & $4.62 \mathrm{E}-06$ \\
\hline \multirow{2}{*}{$F_{13}$} & Ortalama & $2.02 \mathrm{E}-02$ & 1.43E-02 & 2.19E-02 & $1.84 \mathrm{E}-02$ & $2.01 \mathrm{E}-02$ & 1.93E-02 & $1.37 \mathrm{E}-01$ & $1.04 \mathrm{E}-02$ \\
\hline & S. S. & $1.57 \mathrm{E}-02$ & $4.26 \mathrm{E}-03$ & $1.67 \mathrm{E}-02$ & $1.24 \mathrm{E}-02$ & $1.69 \mathrm{E}-02$ & 5.57E-03 & 7.87E-02 & 4.07E-03 \\
\hline \multirow{2}{*}{$F_{14}$} & Ortalama & $1.18 \mathrm{E}+01$ & $4.73 \mathrm{E}+01$ & $4.73 \mathrm{E}+01$ & $6.36 \mathrm{E}-05$ & $2.36 \mathrm{E}+01$ & $6.36 \mathrm{E}-05$ & $4.42 \mathrm{E}+02$ & $1.16 \mathrm{E}-04$ \\
\hline & S. S. & $3.61 \mathrm{E}+01$ & $5.80 \mathrm{E}+01$ & $5.80 \mathrm{E}+01$ & $0.00 \mathrm{E}+00$ & $4.73 \mathrm{E}+01$ & $0.00 E+00$ & $1.31 \mathrm{E}+02$ & 4.67E-05 \\
\hline \multirow{2}{*}{$F_{15}$} & Ortalama & $7.11 \mathrm{E}-16$ & $0.00 E+00$ & $0.00 E+00$ & $0.00 \mathrm{E}+00$ & $0.00 \mathrm{E}+00$ & $0.00 E+00$ & $1.92 \mathrm{E}+00$ & $1.73 \mathrm{E}-03$ \\
\hline & S. S. & $1.45 \mathrm{E}-15$ & $0.00 \mathrm{E}+00$ & $0.00 E+00$ & $0.00 \mathrm{E}+00$ & $0.00 \mathrm{E}+00$ & $0.00 \mathrm{E}+00$ & $1.64 \mathrm{E}+00$ & $5.92 \mathrm{E}-04$ \\
\hline \multirow{2}{*}{$F_{16}$} & Ortalama & 9.42E-32 & $9.42 \mathrm{E}-32$ & 9.42E-32 & 9.42E-32 & $9.42 E-32$ & 9.42E-32 & $2.02 \mathrm{E}+00$ & $5.02 \mathrm{E}-07$ \\
\hline & S. S. & $3.34 \mathrm{E}-47$ & $0.00 \mathrm{E}+00$ & $0.00 E+00$ & $0.00 \mathrm{E}+00$ & $0.00 \mathrm{E}+00$ & $0.00 \mathrm{E}+00$ & $1.68 \mathrm{E}+00$ & $4.84 \mathrm{E}-07$ \\
\hline \multirow{2}{*}{$F_{17}$} & Ortalama & $3.66 \mathrm{E}-04$ & $1.34 \mathrm{E}-32$ & $1.34 \mathrm{E}-32$ & $1.34 \mathrm{E}-32$ & $1.34 \mathrm{E}-32$ & 1.34E-32 & $7.35 \mathrm{E}+03$ & $3.57 \mathrm{E}-06$ \\
\hline & S. S. & $2.01 \mathrm{E}-03$ & $0.00 E+00$ & $0.00 E+00$ & $0.00 \mathrm{E}+00$ & $0.00 E+00$ & $0.00 E+00$ & $7.05 E+03$ & $2.86 \mathrm{E}-06$ \\
\hline \multirow{2}{*}{$F_{18}$} & Ortalama & $1.30 \mathrm{E}-09$ & $3.46 \mathrm{E}-19$ & $1.56 \mathrm{E}-17$ & $4.44 \mathrm{E}-16$ & $3.82 \mathrm{E}-11$ & $4.73 \mathrm{E}-12$ & $4.26 \mathrm{E}-02$ & 6.33E-05 \\
\hline & S. S. & 7.14E-09 & $6.93 E-19$ & $2.86 \mathrm{E}-17$ & $8.88 \mathrm{E}-16$ & $7.64 \mathrm{E}-11$ & $9.47 \mathrm{E}-12$ & $4.61 \mathrm{E}-02$ & $5.84 \mathrm{E}-05$ \\
\hline \multirow{2}{*}{$F_{19}$} & Ortalama & 7.32E-03 & $1.34 \mathrm{E}-31$ & $1.34 \mathrm{E}-31$ & $1.34 \mathrm{E}-31$ & 1.34E-31 & 1.34E-31 & $1.23 \mathrm{E}-01$ & 5.87E-05 \\
\hline & S. S. & $2.79 \mathrm{E}-02$ & $0.00 E+00$ & $0.00 E+00$ & $0.00 \mathrm{E}+00$ & $0.00 \mathrm{E}+00$ & $0.00 E+00$ & $1.16 \mathrm{E}-01$ & $6.30 \mathrm{E}-05$ \\
\hline \multirow{2}{*}{$F_{20}$} & Ortalama & $0.00 \mathrm{E}+00$ & $0.00 E+00$ & $0.00 E+00$ & $0.00 \mathrm{E}+00$ & $0.00 \mathrm{E}+00$ & $0.00 \mathrm{E}+00$ & $1.66 \mathrm{E}-01$ & $1.25 \mathrm{E}-02$ \\
\hline & S. S. & $0.00 \mathrm{E}+00$ & $0.00 \mathrm{E}+00$ & $0.00 \mathrm{E}+00$ & $0.00 \mathrm{E}+00$ & $0.00 \mathrm{E}+00$ & $0.00 \mathrm{E}+00$ & $1.87 \mathrm{E}-01$ & $2.43 \mathrm{E}-03$ \\
\hline & Ortalama & 7.16E-03 & $9.71 \mathrm{E}-03$ & $9.71 \mathrm{E}-03$ & $9.71 \mathrm{E}-03$ & $9.71 \mathrm{E}-03$ & $9.71 \mathrm{E}-03$ & $1.68 \mathrm{E}-01$ & $2.62 \mathrm{E}-02$ \\
\hline$F_{21}$ & S. S. & $9.38 \mathrm{E}-10$ & $1.00 \mathrm{E}-10$ & $3.20 \mathrm{E}-10$ & $1.51 \mathrm{E}-10$ & $2.02 \mathrm{E}-10$ & $2.24 \mathrm{E}-10$ & $1.30 \mathrm{E}-01$ & $1.34 \mathrm{E}-02$ \\
\hline & Ortalama & $-7.83 E+01$ & $-7.83 E+01$ & $-7.83 E+01$ & $-7.83 E+01$ & $-7.83 E+01$ & $-7.83 E+01$ & $-7.44 \mathrm{E}+01$ & $-7.83 E+01$ \\
\hline$F_{22}$ & S. S. & $1.77 \mathrm{E}-14$ & $0.00 E+00$ & $0.00 E+00$ & $0.00 \mathrm{E}+00$ & $0.00 E+00$ & $0.00 E+00$ & $7.33 \mathrm{E}+00$ & $3.72 \mathrm{E}-08$ \\
\hline$F_{2}$ & Ortalama & $-4.69 E+00$ & $2.27 \mathrm{E}-22$ & $2.20 \mathrm{E}-19$ & $5.83 \mathrm{E}-18$ & $4.41 \mathrm{E}-19$ & $1.10 \mathrm{E}-19$ & $1.79 \mathrm{E}-04$ & $1.65 \mathrm{E}-17$ \\
\hline$F_{23}$ & S. S. & $6.70 \mathrm{E}-02$ & 1.13E-22 & $2.70 \mathrm{E}-19$ & $1.08 \mathrm{E}-17$ & $2.20 \mathrm{E}-19$ & $2.20 \mathrm{E}-19$ & $2.51 \mathrm{E}-04$ & $1.32 \mathrm{E}-17$ \\
\hline & Ortalama & $1.82 \mathrm{E}-26$ & $4.04 \mathrm{E}-31$ & $3.45 \mathrm{E}-31$ & $3.62 \mathrm{E}-31$ & $2.05 \mathrm{E}-30$ & $4.55 \mathrm{E}-30$ & $4.18 \mathrm{E}+00$ & $1.33 \mathrm{E}-01$ \\
\hline$F_{24}$ & S. S. & $8.99 E-26$ & $2.23 E-31$ & 1.83E-31 & $1.15 \mathrm{E}-31$ & $2.18 \mathrm{E}-30$ & $8.31 \mathrm{E}-30$ & $5.12 \mathrm{E}+00$ & $2.66 \mathrm{E}-01$ \\
\hline
\end{tabular}


Tablo 2. 10-boyutlu test fonksiyonları için elde edilen sonuçlar

\begin{tabular}{|c|c|c|c|c|c|c|c|c|c|}
\hline \multirow[t]{2}{*}{$F$} & İndeks & TSA & CTSA-1 & CTSA-2 & CTSA-3 & CTSA-4 & CTSA-5 & $\mathrm{DE}$ & HS \\
\hline & En iyi & $3.69 \mathrm{E}-241$ & $2.06 \mathrm{E}-239$ & $3.83 \mathrm{E}-241$ & $2.16 \mathrm{E}-240$ & $2.40 \mathrm{E}-244$ & $1.86 \mathrm{E}-238$ & $1.77 \mathrm{E}+002$ & $1.33 \mathrm{E}-005$ \\
\hline \multirow[t]{3}{*}{$F_{1}$} & Ortalama & 1.73E-237 & 7.71E-236 & 2.68E-234 & $8.29 E-236$ & 3.33E-235 & $1.35 \mathrm{E}-234$ & $1.26 \mathrm{E}+003$ & $3.66 \mathrm{E}-005$ \\
\hline & S. S. & 1.73E-238 & $2.61 \mathrm{E}-237$ & $7.68 \mathrm{E}-235$ & 2.19E-237 & $9.23 \mathrm{E}-237$ & $7.45 \mathrm{E}-236$ & $1.44 \mathrm{E}+003$ & $2.48 \mathrm{E}-005$ \\
\hline & En iyi & $1.43 \mathrm{E}-237$ & $1.99 \mathrm{E}-237$ & $3.94 \mathrm{E}-237$ & $1.32 \mathrm{E}-237$ & $7.88 E-239$ & $2.65 \mathrm{E}-238$ & $1.50 \mathrm{E}+005$ & 4.24E-001 \\
\hline \multirow[t]{3}{*}{$F_{2}$} & Ortalama & 2.29E-231 & $1.46 \mathrm{E}-234$ & 7.31E-230 & $2.09 \mathrm{E}-232$ & 1.32E-235 & $4.36 \mathrm{E}-233$ & $1.69 \mathrm{E}+006$ & $4.26 \mathrm{E}-001$ \\
\hline & S. S. & 8.39E-232 & $3.16 \mathrm{E}-235$ & $6.34 \mathrm{E}-232$ & $3.45 \mathrm{E}-234$ & 7.54E-237 & $3.28 \mathrm{E}-236$ & $2.25 \mathrm{E}+006$ & $5.32 \mathrm{E}+000$ \\
\hline & En iyi & $6.96 \mathrm{E}-242$ & $2.44 \mathrm{E}-242$ & $1.19 \mathrm{E}-240$ & $7.16 \mathrm{E}-241$ & $6.91 \mathrm{E}-243$ & $6.21 E-243$ & $5.19 \mathrm{E}+000$ & $7.35 \mathrm{E}-007$ \\
\hline \multirow[t]{3}{*}{$F_{3}$} & Ortalama & $4.42 \mathrm{E}-238$ & $1.06 \mathrm{E}-238$ & 8.03E-238 & 7.92E-238 & $1.11 \mathrm{E}-236$ & $5.86 \mathrm{E}-237$ & $4.31 \mathrm{E}+001$ & $1.62 \mathrm{E}-006$ \\
\hline & S. S. & $5.51 \mathrm{E}-239$ & $6.12 \mathrm{E}-240$ & 3.14E-239 & $5.54 \mathrm{E}-240$ & $2.17 \mathrm{E}-238$ & 4.15E-239 & $3.62 \mathrm{E}+001$ & $9.21 \mathrm{E}-007$ \\
\hline & En iyi & $0.00 \mathrm{E}+00$ & $0.00 E+00$ & $0.00 E+00$ & $0.00 \mathrm{E}+00$ & $0.00 \mathrm{E}+00$ & $0.00 E+00$ & $4.26 \mathrm{E}-001$ & 3.97E-009 \\
\hline \multirow{3}{*}{$F_{4}$} & Ortalama & $0.00 \mathrm{E}+00$ & $0.00 E+00$ & $0.00 E+00$ & $0.00 E+00$ & $0.00 \mathrm{E}+00$ & $0.00 E+00$ & $5.46 \mathrm{E}+002$ & 6.65E-009 \\
\hline & S. S. & $0.00 \mathrm{E}+00$ & $0.00 E+00$ & $0.00 E+00$ & $0.00 \mathrm{E}+00$ & $0.00 \mathrm{E}+00$ & $0.00 E+00$ & $6.14 \mathrm{E}+002$ & $3.50 \mathrm{E}-009$ \\
\hline & En iyi & 4.63E-154 & $1.58 \mathrm{E}-152$ & 7.36E-153 & $9.61 \mathrm{E}-154$ & 1.77E-154 & $4.78 \mathrm{E}-154$ & $2.14 \mathrm{E}-001$ & 1.07E-003 \\
\hline \multirow[t]{3}{*}{$F_{5}$} & Ortalama & 8.36E-152 & $3.42 \mathrm{E}-150$ & 3.20E-151 & $3.84 \mathrm{E}-150$ & $1.55 \mathrm{E}-150$ & $2.43 \mathrm{E}-149$ & $3.41 \mathrm{E}+000$ & $1.18 \mathrm{E}-003$ \\
\hline & S. S. & 1.52E-151 & $6.34 \mathrm{E}-150$ & 3.47E-151 & $7.56 \mathrm{E}-150$ & $2.55 \mathrm{E}-150$ & $4.86 \mathrm{E}-149$ & $2.56 \mathrm{E}+000$ & 8.91E-005 \\
\hline & En iyi & $8.14 \mathrm{E}-37$ & $3.74 \mathrm{E}-37$ & $4.63 \mathrm{E}-36$ & $9.42 \mathrm{E}-36$ & 3.52E-38 & $3.86 \mathrm{E}-36$ & $1.31 \mathrm{E}+01$ & $9.33 \mathrm{E}-03$ \\
\hline \multirow[t]{3}{*}{$F_{6}$} & Ortalama & $2.48 \mathrm{E}-33$ & 8.72E-34 & $2.45 \mathrm{E}-33$ & $6.72 \mathrm{E}-34$ & $3.81 \mathrm{E}-33$ & 1.10E-34 & $2.22 \mathrm{E}+01$ & $1.05 \mathrm{E}-02$ \\
\hline & S. S. & $4.89 \mathrm{E}-33$ & $1.41 \mathrm{E}-33$ & $4.16 \mathrm{E}-33$ & $1.24 \mathrm{E}-33$ & $6.76 \mathrm{E}-33$ & $1.22 \mathrm{E}-34$ & $7.17 \mathrm{E}+00$ & $9.20 \mathrm{E}-04$ \\
\hline & En iyi & $0.00 \mathrm{E}+00$ & $0.00 E+00$ & $0.00 E+00$ & $0.00 E+00$ & $0.00 E+00$ & $0.00 E+00$ & $1.64 \mathrm{E}+02$ & $0.00 \mathrm{E}+00$ \\
\hline \multirow[t]{3}{*}{$F_{7}$} & Ortalama & $0.00 \mathrm{E}+00$ & $0.00 E+00$ & $0.00 E+00$ & $0.00 E+00$ & $0.00 \mathrm{E}+00$ & $0.00 E+00$ & $6.95 \mathrm{E}+02$ & $0.00 \mathrm{E}+00$ \\
\hline & S. S. & $0.00 E+00$ & $0.00 E+00$ & $0.00 E+00$ & $0.00 E+00$ & $0.00 E+00$ & $0.00 E+00$ & $8.87 \mathrm{E}+02$ & $0.00 \mathrm{E}+00$ \\
\hline & En iyi & $0.00 \mathrm{E}+00$ & $0.00 E+00$ & $0.00 E+00$ & $0.00 E+00$ & $0.00 \mathrm{E}+00$ & $0.00 E+00$ & $9.71 \mathrm{E}-04$ & $5.58 \mathrm{E}-17$ \\
\hline$F_{8}$ & Ortalama & $0.00 \mathrm{E}+00$ & $0.00 E+00$ & $0.00 E+00$ & $0.00 E+00$ & $0.00 \mathrm{E}+00$ & $0.00 E+00$ & $1.18 \mathrm{E}-01$ & $1.32 \mathrm{E}-16$ \\
\hline & S. S. & $0.00 \mathrm{E}+00$ & $0.00 E+00$ & $0.00 E+00$ & $0.00 \mathrm{E}+00$ & $0.00 \mathrm{E}+00$ & $0.00 E+00$ & $1.31 \mathrm{E}-01$ & 8.67E-17 \\
\hline & En iyi & $5.66 \mathrm{E}-04$ & $2.27 \mathrm{E}-04$ & $4.05 \mathrm{E}-04$ & 1.97E-04 & $4.20 \mathrm{E}-04$ & 3.97E-04 & $6.30 \mathrm{E}-03$ & 8.73E-04 \\
\hline$F_{9}$ & Ortalama & $9.86 \mathrm{E}-04$ & 8.51E-04 & $6.58 \mathrm{E}-04$ & $9.73 \mathrm{E}-04$ & $9.34 \mathrm{E}-04$ & 7.81E-04 & 5.57E-02 & 2.33E-03 \\
\hline & S. S. & $4.57 \mathrm{E}-04$ & 3.31E-04 & $2.95 \mathrm{E}-04$ & $5.67 \mathrm{E}-04$ & $4.49 \mathrm{E}-04$ & $2.90 \mathrm{E}-04$ & $6.95 \mathrm{E}-02$ & $1.23 \mathrm{E}-03$ \\
\hline & En iyi & $1.86 \mathrm{E}-01$ & $1.50 \mathrm{E}-01$ & 1.80E-03 & $6.46 \mathrm{E}-03$ & $1.71 \mathrm{E}-01$ & $1.91 \mathrm{E}-01$ & $1.95 E+03$ & $5.53 \mathrm{E}-01$ \\
\hline$F_{10}$ & Ortalama & $1.29 \mathrm{E}+00$ & $1.84 \mathrm{E}+00$ & $9.82 \mathrm{E}-01$ & 8.60E-01 & $1.11 \mathrm{E}+00$ & 3.95E-01 & $3.00 \mathrm{E}+03$ & $3.39 E+00$ \\
\hline & S.S. & $1.41 \mathrm{E}+00$ & $1.77 \mathrm{E}+00$ & $1.53 \mathrm{E}+00$ & $1.65 \mathrm{E}+00$ & $1.58 \mathrm{E}+00$ & 2.06E-01 & $9.84 \mathrm{E}+02$ & $2.02 \mathrm{E}+00$ \\
\hline & En iyi & $9.94 \mathrm{E}-01$ & $0.00 E+00$ & $0.00 \mathrm{E}+00$ & $9.94 \mathrm{E}-01$ & $0.00 \mathrm{E}+00$ & $0.00 \mathrm{E}+00$ & $6.26 \mathrm{E}+00$ & $1.46 \mathrm{E}-05$ \\
\hline$F_{11}$ & Ortalama & $2.18 \mathrm{E}+00$ & $2.18 \mathrm{E}+00$ & $1.19 \mathrm{E}+00$ & $2.98 \mathrm{E}+00$ & $1.39 \mathrm{E}+00$ & 7.95E-01 & $1.28 \mathrm{E}+01$ & $3.69 \mathrm{E}+00$ \\
\hline & S. S. & $1.16 \mathrm{E}+00$ & $1.46 \mathrm{E}+00$ & $1.16 \mathrm{E}+00$ & $2.26 \mathrm{E}+00$ & 7.95E-01 & 3.97E-01 & $4.60 \mathrm{E}+00$ & $1.79 E+00$ \\
\hline & En iyi & $2.00 \mathrm{E}+00$ & $2.00 \mathrm{E}+00$ & $3.00 \mathrm{E}+00$ & $3.00 \mathrm{E}+00$ & $1.00 \mathrm{E}+00$ & $2.00 \mathrm{E}+00$ & $1.00 \mathrm{E}+01$ & $8.90 \mathrm{E}+00$ \\
\hline$F_{12}$ & Ortalama & $3.20 \mathrm{E}+00$ & $3.20 \mathrm{E}+00$ & $9.00 \mathrm{E}+00$ & $4.60 \mathrm{E}+00$ & $2.60 \mathrm{E}+00$ & $3.00 \mathrm{E}+00$ & $1.42 \mathrm{E}+01$ & $4.55 \mathrm{E}+00$ \\
\hline & S. S. & $7.48 \mathrm{E}-01$ & $7.48 \mathrm{E}-01$ & $1.15 \mathrm{E}+01$ & $1.01 \mathrm{E}+00$ & $1.01 \mathrm{E}+00$ & $6.32 \mathrm{E}-01$ & $5.16 \mathrm{E}+00$ & $1.55 E+00$ \\
\hline & En iyi & $1.17 \mathrm{E}-02$ & $1.11 \mathrm{E}-16$ & $9.85 \mathrm{E}-03$ & $1.09 \mathrm{E}-02$ & $1.23 \mathrm{E}-02$ & $0.00 \mathrm{E}+00$ & $2.29 \mathrm{E}+00$ & $1.24 \mathrm{E}-02$ \\
\hline$F_{13}$ & Ortalama & $1.56 \mathrm{E}-02$ & $1.91 \mathrm{E}-02$ & 3.30E-02 & $2.05 \mathrm{E}-02$ & $1.83 \mathrm{E}-02$ & $2.57 \mathrm{E}-02$ & $1.60 \mathrm{E}+01$ & $2.23 \mathrm{E}-02$ \\
\hline & S. S. & 3.74E-03 & $1.24 \mathrm{E}-02$ & $1.95 \mathrm{E}-02$ & 6.33E-03 & 4.78E-03 & $1.52 \mathrm{E}-02$ & $1.24 \mathrm{E}+01$ & 7.36E-03 \\
\hline & En iyi & $1.27 \mathrm{E}-04$ & $1.18 \mathrm{E}+02$ & 1.27E-04 & $1.27 \mathrm{E}-04$ & $1.18 \mathrm{E}+02$ & 1.27E-04 & $9.46 \mathrm{E}+02$ & $1.74 \mathrm{E}-04$ \\
\hline$F_{14}$ & Ortalama & $2.13 \mathrm{E}+02$ & $2.13 E+02$ & $7.10 \mathrm{E}+01$ & 1.27E-04 & $2.09 \mathrm{E}+02$ & $1.85 E+02$ & $1.14 \mathrm{E}+03$ & $2.68 \mathrm{E}-04$ \\
\hline & S. S. & $1.16 \mathrm{E}+02$ & $4.73 E+01$ & $9.47 \mathrm{E}+01$ & $0.00 E+00$ & $8.24 \mathrm{E}+01$ & $1.15 E+02$ & $1.72 \mathrm{E}+02$ & 7.05E-05 \\
\hline & En iyi & $0.00 \mathrm{E}+00$ & $0.00 E+00$ & $0.00 \mathrm{E}+00$ & $0.00 \mathrm{E}+00$ & $0.00 \mathrm{E}+00$ & $0.00 \mathrm{E}+00$ & $4.35 \mathrm{E}+00$ & $2.23 \mathrm{E}-03$ \\
\hline$F_{15}$ & Ortalama & 8.88E-16 & 8.88E-16 & 8.88E-16 & 8.88E-16 & 8.88E-16 & 8.88E-16 & $8.99 \mathrm{E}+00$ & $2.40 \mathrm{E}-03$ \\
\hline & S. S. & 1.08E-15 & $1.08 \mathrm{E}-15$ & $1.08 \mathrm{E}-15$ & $1.08 \mathrm{E}-15$ & $1.08 E-15$ & $1.08 E-15$ & $3.16 \mathrm{E}+00$ & $1.63 \mathrm{E}-04$ \\
\hline & En iyi & 4.71E-32 & $4.71 \mathrm{E}-32$ & 4.71E-32 & 4.71E-32 & 4.71E-32 & 4.71E-32 & $2.70 \mathrm{E}+00$ & $1.72 \mathrm{E}-07$ \\
\hline$F_{16}$ & Ortalama & $2.48 \mathrm{E}-01$ & $4.71 \mathrm{E}-32$ & 4.71E-32 & $4.71 \mathrm{E}-32$ & 4.71E-32 & 4.71E-32 & $4.66 \mathrm{E}+05$ & $5.76 \mathrm{E}-07$ \\
\hline & S. S. & 4.97E-01 & $0.00 E+00$ & $0.00 \mathrm{E}+00$ & $0.00 E+00$ & $0.00 \mathrm{E}+00$ & $0.00 \mathrm{E}+00$ & $6.17 \mathrm{E}+00$ & $3.80 \mathrm{E}-07$ \\
\hline & En iyi & $1.34 \mathrm{E}-32$ & $1.34 \mathrm{E}-32$ & $1.34 \mathrm{E}-32$ & $1.34 \mathrm{E}-32$ & $1.34 \mathrm{E}-32$ & $1.34 \mathrm{E}-32$ & $6.87 \mathrm{E}+03$ & $4.10 \mathrm{E}-06$ \\
\hline$F_{17}$ & Ortalama & $1.39 \mathrm{E}-32$ & $1.34 \mathrm{E}-32$ & $1.70 \mathrm{E}-32$ & $1.34 \mathrm{E}-32$ & $1.34 \mathrm{E}-32$ & $1.34 \mathrm{E}-32$ & $6.20 \mathrm{E}+05$ & $2.10 \mathrm{E}-05$ \\
\hline & S. S. & $8.42 \mathrm{E}-34$ & $0.00 E+00$ & $5.00 \mathrm{E}-33$ & $0.00 E+00$ & $0.00 E+00$ & $0.00 E+00$ & $4.30 \mathrm{E}+05$ & $2.22 \mathrm{E}-05$ \\
\hline & En iyi & $2.61 \mathrm{E}-261$ & $7.05 E-308$ & 4.49E-322 & $7.46 \mathrm{E}-264$ & $5.04 \mathrm{E}-290$ & $7.25 \mathrm{E}-317$ & 2.93E-002 & 5.85E-005 \\
\hline$F_{18}$ & Ortalama & 2.35E-016 & 2.38E-124 & $2.49 \mathrm{E}-016$ & $1.25 \mathrm{E}-016$ & $1.66 \mathrm{E}-016$ & $1.74 \mathrm{E}-144$ & 6.71E-001 & $2.34 \mathrm{E}-004$ \\
\hline & S. S. & 4.70E-016 & $4.76 \mathrm{E}-124$ & 4.98E-016 & $2.42 \mathrm{E}-016$ & 3.33E-016 & $3.44 \mathrm{E}-144$ & $6.52 \mathrm{E}-001$ & $2.06 \mathrm{E}-004$ \\
\hline & En iyi & $1.34 \mathrm{E}-31$ & $1.34 \mathrm{E}-31$ & $1.34 \mathrm{E}-31$ & $1.34 \mathrm{E}-31$ & $1.34 \mathrm{E}-31$ & 1.34E-31 & $3.47 \mathrm{E}+00$ & $6.44 \mathrm{E}-06$ \\
\hline$F_{19}$ & Ortalama & $1.44 \mathrm{E}-31$ & $1.37 \mathrm{E}-31$ & $1.34 \mathrm{E}-31$ & $1.34 \mathrm{E}-31$ & $1.34 \mathrm{E}-31$ & 4.39E-02 & $1.14 \mathrm{E}+01$ & 8.89E-05 \\
\hline & S. S. & $1.97 \mathrm{E}-32$ & $4.93 \mathrm{E}-33$ & $0.00 E+00$ & $0.00 \mathrm{E}+00$ & $0.00 \mathrm{E}+00$ & $5.38 \mathrm{E}-02$ & $1.07 \mathrm{E}+01$ & $6.13 \mathrm{E}-05$ \\
\hline & En iyi & $0.00 \mathrm{E}+00$ & $0.00 E+00$ & $0.00 E+00$ & $0.00 \mathrm{E}+00$ & $0.00 \mathrm{E}+00$ & $0.00 \mathrm{E}+00$ & $9.07 \mathrm{E}-01$ & $2.50 \mathrm{E}-02$ \\
\hline$F_{20}$ & Ortalama & $0.00 E+00$ & $0.00 E+00$ & $0.00 E+00$ & $0.00 E+00$ & $0.00 E+00$ & $0.00 E+00$ & $3.03 E+00$ & 3.31E-02 \\
\hline & S. S. & $0.00 E+00$ & $0.00 E+00$ & $0.00 E+00$ & $0.00 E+00$ & $0.00 E+00$ & $0.00 E+00$ & $1.25 \mathrm{E}+00$ & $9.75 \mathrm{E}-03$ \\
\hline & En iyi & 9.71E-03 & 9.71E-03 & 9.71E-03 & 9.71E-03 & 9.71E-03 & 9.71E-03 & $1.26 \mathrm{E}-01$ & 3.72E-02 \\
\hline$F_{21}$ & Ortalama & 9.71E-03 & 9.71E-03 & 9.71E-03 & 9.71E-03 & 9.71E-03 & 9.71E-03 & $3.00 \mathrm{E}-01$ & 8.08E-02 \\
\hline & S. S. & $4.74 \mathrm{E}-11$ & $2.09 \mathrm{E}-10$ & $3.42 \mathrm{E}-10$ & $7.14 \mathrm{E}-10$ & $4.05 \mathrm{E}-10$ & 8.55E-09 & $1.23 \mathrm{E}-01$ & $3.66 \mathrm{E}-02$ \\
\hline & En iyi & $-7.83 E+01$ & $-7.83 E+01$ & $-7.83 E+01$ & $-7.83 E+01$ & $-7.83 E+01$ & $-7.83 E+01$ & $-7.27 \mathrm{E}+01$ & $-7.83 E+01$ \\
\hline$F_{22}$ & Ortalama & $-7.72 \mathrm{E}+01$ & $-7.83 E+01$ & $-7.72 \mathrm{E}+01$ & $-7.09 \mathrm{E}+01$ & $-7.72 \mathrm{E}+01$ & $-7.66 \mathrm{E}+01$ & $-6.76 \mathrm{E}+01$ & $-7.84 E+01$ \\
\hline & S. S. & $1.38 \mathrm{E}+00$ & $0.00 E+00$ & $1.38 \mathrm{E}+00$ & $2.26 \mathrm{E}+00$ & $1.38 \mathrm{E}+00$ & $1.38 \mathrm{E}+00$ & $3.44 \mathrm{E}+00$ & $1.50 \mathrm{E}-07$ \\
\hline & En iyi & $2.04 \mathrm{E}-29$ & $2.56 \mathrm{E}-77$ & $2.04 \mathrm{E}-29$ & $5.51 \mathrm{E}-19$ & $2.84 \mathrm{E}-22$ & $2.04 \mathrm{E}-29$ & $1.24 \mathrm{E}-16$ & $5.51 \mathrm{E}-19$ \\
\hline$F_{23}$ & Ortalama & $3.31 \mathrm{E}-19$ & 1.13E-22 & $2.20 \mathrm{E}-19$ & $1.13 \mathrm{E}-17$ & $2.20 \mathrm{E}-19$ & $2.20 \mathrm{E}-19$ & $2.11 \mathrm{E}-05$ & $9.35 \mathrm{E}-18$ \\
\hline & S. S. & $2.70 \mathrm{E}-19$ & $1.39 \mathrm{E}-22$ & $2.70 \mathrm{E}-19$ & $1.32 \mathrm{E}-17$ & $2.70 \mathrm{E}-19$ & $2.70 \mathrm{E}-19$ & $4.09 \mathrm{E}-05$ & $1.24 \mathrm{E}-17$ \\
\hline & En iyi & $7.56 \mathrm{E}-30$ & 7.14E-31 & $2.81 \mathrm{E}-30$ & $1.36 \mathrm{E}-30$ & $1.51 \mathrm{E}-30$ & $2.85 \mathrm{E}-30$ & $1.24 \mathrm{E}+02$ & 2.99E-04 \\
\hline$F_{24}$ & Ortalama & $5.33 \mathrm{E}-01$ & 4.33E-01 & $2.66 \mathrm{E}-01$ & 1.33E-01 & $2.66 \mathrm{E}-01$ & $4.00 \mathrm{E}-01$ & $3.27 \mathrm{E}+02$ & $4.44 \mathrm{E}-01$ \\
\hline & S. S. & $2.66 \mathrm{E}-01$ & $2.90 \mathrm{E}-01$ & $3.26 \mathrm{E}-01$ & $2.66 \mathrm{E}-01$ & $3.26 \mathrm{E}-01$ & $3.26 \mathrm{E}-01$ & $1.85 \mathrm{E}+02$ & $3.14 \mathrm{E}-01$ \\
\hline
\end{tabular}


Tablo 3. 100-boyutlu test fonksiyonları için elde edilen sonuclar

\begin{tabular}{|c|c|c|c|c|c|c|c|c|c|}
\hline \multirow[t]{2}{*}{$F$} & İndeks & TSA & CTSA-1 & CTSA-2 & CTSA-3 & CTSA-4 & CTSA-5 & $\mathrm{DE}$ & HS \\
\hline & En iyi & $1.37 \mathrm{E}-11$ & $4.97 \mathrm{E}-12$ & $4.32 \mathrm{E}-11$ & 3.02E-11 & $1.24 \mathrm{E}-12$ & $9.22 \mathrm{E}-12$ & $2.26 \mathrm{E}+04$ & $7.09 E+01$ \\
\hline \multirow[t]{3}{*}{$F_{1}$} & Ortalama & 3.99E-11 & $1.36 \mathrm{E}-10$ & $7.62 \mathrm{E}-11$ & $8.41 \mathrm{E}-11$ & 1.33E-11 & $2.53 \mathrm{E}-11$ & $2.74 \mathrm{E}+04$ & $8.21 \mathrm{E}+01$ \\
\hline & S. S. & $2.97 \mathrm{E}-11$ & $1.57 \mathrm{E}-10$ & $2.34 \mathrm{E}-11$ & $6.28 \mathrm{E}-11$ & $8.56 \mathrm{E}-12$ & $1.16 \mathrm{E}-11$ & $4.08 \mathrm{E}+03$ & $1.07 \mathrm{E}+01$ \\
\hline & En iyi & $3.96 \mathrm{E}-09$ & $3.60 \mathrm{E}-09$ & $2.98 \mathrm{E}-09$ & $8.61 \mathrm{E}-09$ & 2.83E-09 & $1.90 \mathrm{E}-09$ & $3.12 \mathrm{E}+08$ & $8.34 \mathrm{E}+04$ \\
\hline \multirow[t]{3}{*}{$F_{2}$} & Ortalama & 6.68E-09 & 7.61E-09 & $1.00 \mathrm{E}-08$ & 7.29E-09 & $7.29 \mathrm{E}-09$ & 5.37E-09 & $5.75 E+08$ & $9.97 \mathrm{E}+04$ \\
\hline & S. S. & 2.14E-09 & 2.84E-09 & 5.05E-09 & $5.44 \mathrm{E}-09$ & 5.44E-09 & $2.74 \mathrm{E}-09$ & $2.42 \mathrm{E}+08$ & $1.50 \mathrm{E}+04$ \\
\hline & En iyi & $2.85 \mathrm{E}-12$ & $1.72 \mathrm{E}-12$ & $3.24 \mathrm{E}-12$ & $1.84 \mathrm{E}-12$ & $1.45 \mathrm{E}-12$ & $1.35 E-12$ & $3.78 \mathrm{E}+03$ & $9.81 E+00$ \\
\hline \multirow[t]{3}{*}{$F_{3}$} & Ortalama & $3.55 E-12$ & 7.35E-12 & $6.21 \mathrm{E}-12$ & $5.32 \mathrm{E}-12$ & $5.41 \mathrm{E}-12$ & $7.65 \mathrm{E}-12$ & $5.03 E+03$ & $1.25 \mathrm{E}+01$ \\
\hline & S. S. & $7.97 \mathrm{E}-13$ & $5.80 \mathrm{E}-12$ & $3.03 \mathrm{E}-12$ & $3.98 \mathrm{E}-12$ & $2.81 \mathrm{E}-12$ & $5.61 \mathrm{E}-12$ & $1.39 \mathrm{E}+03$ & $2.80 \mathrm{E}+00$ \\
\hline & En iyi & $2.01 \mathrm{E}+01$ & $2.49 \mathrm{E}+01$ & $1.68 \mathrm{E}+02$ & $2.78 \mathrm{E}+02$ & $2.20 \mathrm{E}+01$ & $9.30 \mathrm{E}+00$ & $1.85 E+26$ & $2.56 \mathrm{E}+02$ \\
\hline \multirow[t]{3}{*}{$F_{4}$} & Ortalama & $5.09 \mathrm{E}+02$ & $7.03 E+02$ & $4.09 E+02$ & $4.39 \mathrm{E}+02$ & $1.15 \mathrm{E}+04$ & $1.22 \mathrm{E}+03$ & $1.03 E+30$ & $3.60 \mathrm{E}+03$ \\
\hline & S. S. & $6.45 \mathrm{E}+02$ & $8.98 \mathrm{E}+02$ & $1.73 E+02$ & $1.19 E+02$ & $1.02 \mathrm{E}+04$ & $1.62 E+03$ & $8.74 \mathrm{E}+29$ & $1.56 \mathrm{E}+03$ \\
\hline & En iyi & $2.50 \mathrm{E}-11$ & $2.43 \mathrm{E}-11$ & $3.47 \mathrm{E}-11$ & $3.99 \mathrm{E}-11$ & $1.56 \mathrm{E}-11$ & $2.90 \mathrm{E}-11$ & $7.59 \mathrm{E}+01$ & $2.78 \mathrm{E}+00$ \\
\hline \multirow[t]{3}{*}{$F_{5}$} & Ortalama & $1.11 \mathrm{E}-10$ & $4.08 \mathrm{E}-11$ & $1.15 \mathrm{E}-10$ & $1.47 \mathrm{E}-10$ & 4.63E-11 & $7.16 \mathrm{E}-11$ & $8.76 \mathrm{E}+01$ & $2.92 \mathrm{E}+00$ \\
\hline & S. S. & $6.10 \mathrm{E}-11$ & $1.56 \mathrm{E}-11$ & $6.22 \mathrm{E}-11$ & 8.43E-11 & $2.38 \mathrm{E}-11$ & 4.34E-11 & $9.34 \mathrm{E}+00$ & $1.60 \mathrm{E}-01$ \\
\hline & En iyi & $7.19 \mathrm{E}+01$ & $4.82 \mathrm{E}+01$ & $7.12 \mathrm{E}+01$ & $5.09 \mathrm{E}+01$ & $4.36 \mathrm{E}+01$ & $6.77 \mathrm{E}+01$ & $5.87 \mathrm{E}+01$ & $5.24 \mathrm{E}+01$ \\
\hline \multirow[t]{3}{*}{$F_{6}$} & Ortalama & $7.61 \mathrm{E}+01$ & $6.43 \mathrm{E}+01$ & $7.64 \mathrm{E}+01$ & $5.74 \mathrm{E}+01$ & $4.56 \mathrm{E}+01$ & $7.40 \mathrm{E}+01$ & $6.23 E+01$ & $6.40 \mathrm{E}+01$ \\
\hline & S. S. & $4.94 \mathrm{E}+00$ & $1.27 \mathrm{E}+01$ & $3.83 E+00$ & $6.79 \mathrm{E}+00$ & $2.36 \mathrm{E}+00$ & $4.85 \mathrm{E}+00$ & $4.26 \mathrm{E}+00$ & $5.80 \mathrm{E}+00$ \\
\hline & En iyi & $0.00 E+00$ & $0.00 E+00$ & $0.00 E+00$ & $0.00 E+00$ & $0.00 E+00$ & $0.00 E+00$ & $2.44 \mathrm{E}+04$ & $5.60 \mathrm{E}+01$ \\
\hline \multirow{3}{*}{$F_{7}$} & Ortalama & $0.00 \mathrm{E}+00$ & $0.00 \mathrm{E}+00$ & $0.00 \mathrm{E}+00$ & $0.00 \mathrm{E}+00$ & $0.00 E+00$ & $0.00 \mathrm{E}+00$ & $3.13 \mathrm{E}+04$ & $7.63 \mathrm{E}+01$ \\
\hline & S. S. & $0.00 \mathrm{E}+00$ & $0.00 \mathrm{E}+00$ & $0.00 \mathrm{E}+00$ & $0.00 \mathrm{E}+00$ & $0.00 E+00$ & $0.00 \mathrm{E}+00$ & $5.65 \mathrm{E}+03$ & $1.45 \mathrm{E}+01$ \\
\hline & En iyi & $5.58 \mathrm{E}-12$ & $2.32 \mathrm{E}-11$ & 3.12E-10 & 3.14E-12 & $9.80 \mathrm{E}-12$ & $6.63 \mathrm{E}-11$ & $1.76 \mathrm{E}+01$ & $4.42 \mathrm{E}-04$ \\
\hline$F_{8}$ & Ortalama & 1.33E-10 & $1.90 \mathrm{E}-10$ & $4.04 \mathrm{E}-10$ & $5.87 \mathrm{E}-10$ & $1.79 \mathrm{E}-10$ & $6.25 \mathrm{E}-10$ & $3.52 \mathrm{E}+01$ & 7.09E-04 \\
\hline & S. S. & $1.45 \mathrm{E}-10$ & 1.19E-10 & $6.55 \mathrm{E}-11$ & $4.87 \mathrm{E}-10$ & $1.92 \mathrm{E}-10$ & $7.82 \mathrm{E}-10$ & $1.24 \mathrm{E}+01$ & $3.44 \mathrm{E}-04$ \\
\hline & En iyi & 8.79E-02 & $5.24 \mathrm{E}-02$ & $6.78 \mathrm{E}-02$ & 1.33E-01 & 5.09E-02 & $6.25 \mathrm{E}-02$ & $2.19 \mathrm{E}+01$ & $1.63 \mathrm{E}-01$ \\
\hline$F_{9}$ & Ortalama & $1.13 \mathrm{E}-01$ & 8.45E-02 & 8.55E-02 & $1.42 \mathrm{E}-01$ & $9.13 \mathrm{E}-02$ & $9.37 \mathrm{E}-02$ & $2.67 \mathrm{E}+01$ & 1.89E-01 \\
\hline & S. S. & 2.83E-02 & $2.28 \mathrm{E}-02$ & $1.61 \mathrm{E}-02$ & 1.15E-02 & $3.52 \mathrm{E}-02$ & $2.93 \mathrm{E}-02$ & $4.36 \mathrm{E}+00$ & $2.07 \mathrm{E}-02$ \\
\hline & En iyi & $4.13 \mathrm{E}+01$ & $4.55 \mathrm{E}+01$ & $4.18 \mathrm{E}+01$ & $9.35 \mathrm{E}+01$ & $4.02 \mathrm{E}+01$ & $4.56 \mathrm{E}+01$ & $4.45 \mathrm{E}+05$ & $3.99 E+02$ \\
\hline$F_{10}$ & Ortalama & $9.95 \mathrm{E}+01$ & $7.61 \mathrm{E}+01$ & $6.22 \mathrm{E}+01$ & $1.18 \mathrm{E}+02$ & $8.23 \mathrm{E}+01$ & $6.42 \mathrm{E}+01$ & $5.89 \mathrm{E}+05$ & $4.18 \mathrm{E}+02$ \\
\hline & S. S. & $4.75 \mathrm{E}+01$ & $2.28 \mathrm{E}+01$ & $2.63 \mathrm{E}+01$ & $2.76 \mathrm{E}+01$ & $5.43 \mathrm{E}+01$ & $2.53 \mathrm{E}+01$ & $1.33 \mathrm{E}+05$ & $1.61 \mathrm{E}+02$ \\
\hline & En iyi & $6.16 \mathrm{E}+01$ & $7.66 \mathrm{E}+01$ & $5.56 \mathrm{E}+01$ & $3.99 E+01$ & $5.47 \mathrm{E}+01$ & $7.16 \mathrm{E}+01$ & $2.42 \mathrm{E}+02$ & $8.03 E+01$ \\
\hline$F_{11}$ & Ortalama & $6.73 E+01$ & $8.72 \mathrm{E}+01$ & 1.13E+01 & $7.01 \mathrm{E}+01$ & $6.20 \mathrm{E}+01$ & $7.92 \mathrm{E}+01$ & $3.01 \mathrm{E}+02$ & $9.29 E+01$ \\
\hline & S. S. & $5.39 \mathrm{E}+00$ & $8.52 \mathrm{E}+00$ & $3.15 E+00$ & $2.96 \mathrm{E}+00$ & $8.29 \mathrm{E}+00$ & $7.00 \mathrm{E}+00$ & $7.30 \mathrm{E}+01$ & $4.26 \mathrm{E}+00$ \\
\hline & En iyi & $7.78 \mathrm{E}+02$ & $1.50 \mathrm{E}+02$ & $1.24 \mathrm{E}+02$ & $1.65 E+02$ & $9.04 \mathrm{E}+01$ & $1.34 \mathrm{E}+02$ & $1.60 \mathrm{E}+02$ & $2.47 \mathrm{E}+02$ \\
\hline$F_{12}$ & Ortalama & $5.57 \mathrm{E}+02$ & $2.07 \mathrm{E}+02$ & $1.84 \mathrm{E}+02$ & $2.49 \mathrm{E}+02$ & $1.29 \mathrm{E}+02$ & $1.96 \mathrm{E}+02$ & $1.89 \mathrm{E}+02$ & $2.63 E+02$ \\
\hline & S. S. & $4.16 \mathrm{E}+01$ & $4.99 \mathrm{E}+01$ & $4.26 \mathrm{E}+01$ & $6.92 \mathrm{E}+01$ & $2.81 \mathrm{E}+01$ & $4.41 \mathrm{E}+01$ & $2.29 \mathrm{E}+01$ & $4.74 \mathrm{E}+01$ \\
\hline & En iyi & $9.38 \mathrm{E}-11$ & $3.38 \mathrm{E}-11$ & $3.01 \mathrm{E}-11$ & $9.04 \mathrm{E}-11$ & $9.25 \mathrm{E}-12$ & $1.16 \mathrm{E}-11$ & $1.86 \mathrm{E}+02$ & $1.45 \mathrm{E}+00$ \\
\hline$F_{13}$ & Ortalama & 3.29E-10 & $2.51 \mathrm{E}-10$ & 8.52E-11 & 1.87E-09 & $2.45 \mathrm{E}-10$ & $3.36 \mathrm{E}-10$ & $2.35 \mathrm{E}+02$ & $1.62 \mathrm{E}+00$ \\
\hline & S. S. & $3.28 \mathrm{E}-10$ & $2.54 \mathrm{E}-10$ & 3.89E-11 & 2.20E-09 & $1.67 \mathrm{E}-10$ & $4.39 \mathrm{E}-10$ & $6.76 \mathrm{E}+01$ & $1.26 \mathrm{E}-01$ \\
\hline & En iyi & $3.57 \mathrm{E}+04$ & $4.34 \mathrm{E}+03$ & $2.72 \mathrm{E}+04$ & $3.08 \mathrm{E}+03$ & $4.18 \mathrm{E}+03$ & $2.78 E+03$ & $1.02 \mathrm{E}+04$ & $8.08 \mathrm{E}+03$ \\
\hline$F_{14}$ & Ortalama & $4.27 \mathrm{E}+04$ & $5.10 \mathrm{E}+03$ & $4.05 \mathrm{E}+04$ & $4.03 E+03$ & $7.67 \mathrm{E}+03$ & $4.73 E+03$ & $1.08 \mathrm{E}+04$ & $1.31 \mathrm{E}+04$ \\
\hline & S. S. & $8.30 \mathrm{E}+03$ & $6.73 E+02$ & 1.19E+03 & $1.26 \mathrm{E}+03$ & $2.65 E+03$ & $2.15 \mathrm{E}+03$ & $4.75 E+03$ & $6.03 E+03$ \\
\hline & En iyi & $9.30 \mathrm{E}-07$ & $2.46 \mathrm{E}-06$ & $1.55 \mathrm{E}-06$ & 7.75E-07 & 4.42E-07 & $8.45 \mathrm{E}-07$ & $1.37 \mathrm{E}+01$ & $3.40 \mathrm{E}+00$ \\
\hline$F_{15}$ & Ortalama & $1.47 \mathrm{E}-06$ & $5.24 \mathrm{E}-06$ & $4.01 \mathrm{E}-06$ & 1.01E-06 & $2.90 \mathrm{E}-06$ & $1.06 \mathrm{E}-06$ & $1.58 \mathrm{E}+01$ & $3.80 \mathrm{E}++$ \\
\hline & S. S. & $5.82 \mathrm{E}-07$ & $3.05 \mathrm{E}-06$ & $3.47 \mathrm{E}-06$ & $1.71 \mathrm{E}-07$ & $1.83 \mathrm{E}-06$ & 1.58E-07 & $1.45 \mathrm{E}+00$ & $3.07 \mathrm{E}-01$ \\
\hline & En iyi & $3.85 \mathrm{E}+00$ & $6.74 \mathrm{E}-01$ & 4.18E-01 & 4.04E-01 & $6.76 \mathrm{E}+00$ & $7.31 \mathrm{E}+00$ & $1.06 \mathrm{E}+07$ & $1.77 \mathrm{E}+00$ \\
\hline$F_{16}$ & Ortalama & $8.35 \mathrm{E}+00$ & $5.24 \mathrm{E}+00$ & $1.01 E+00$ & $2.19 \mathrm{E}+00$ & $8.57 \mathrm{E}+00$ & $1.06 \mathrm{E}+01$ & $3.95 \mathrm{E}+07$ & $2.28 \mathrm{E}+00$ \\
\hline & S. S. & $3.20 \mathrm{E}+00$ & $3.88 \mathrm{E}+00$ & 7.73E-01 & $2.05 \mathrm{E}+00$ & $2.07 \mathrm{E}+00$ & $4.10 \mathrm{E}+00$ & $3.03 E+07$ & $3.10 \mathrm{E}+00$ \\
\hline & En iyi & $1.35 \mathrm{E}-01$ & $4.99 \mathrm{E}-02$ & $5.73 E+00$ & $2.29 \mathrm{E}+00$ & 3.79E-02 & $2.48 \mathrm{E}-01$ & $4.17 \mathrm{E}+07$ & $6.96 \mathrm{E}+00$ \\
\hline$F_{17}$ & Ortalama & $7.27 \mathrm{E}+00$ & $1.38 \mathrm{E}+01$ & $1.80 \mathrm{E}+01$ & $5.58 \mathrm{E}+00$ & $1.27 \mathrm{E}+00$ & $3.28 \mathrm{E}+00$ & $2.06 \mathrm{E}+08$ & $1.97 \mathrm{E}+01$ \\
\hline & S. S. & $6.34 \mathrm{E}+00$ & $1.43 \mathrm{E}+01$ & $1.10 \mathrm{E}+01$ & $2.74 \mathrm{E}+00$ & $1.71 \mathrm{E}+00$ & $3.37 \mathrm{E}+00$ & $1.21 \mathrm{E}+08$ & $1.13 \mathrm{E}+01$ \\
\hline & En iyi & $2.60 \mathrm{E}-03$ & 3.81E-07 & $1.73 \mathrm{E}-02$ & $1.26 \mathrm{E}-03$ & $1.09 \mathrm{E}-03$ & $3.40 \mathrm{E}-06$ & $2.67 \mathrm{E}+01$ & $1.35 \mathrm{E}+00$ \\
\hline$F_{18}$ & Ortalama & 7.65E-02 & 1.09E-05 & $1.03 \mathrm{E}+00$ & $1.71 \mathrm{E}+00$ & $1.04 \mathrm{E}-02$ & $3.83 \mathrm{E}-03$ & $2.88 \mathrm{E}+01$ & $1.43 \mathrm{E}+00$ \\
\hline & S. S. & $1.00 \mathrm{E}-02$ & 1.22E-05 & $1.43 \mathrm{E}+00$ & $2.41 \mathrm{E}+00$ & 8.20E-03 & $5.42 \mathrm{E}-03$ & $2.42 \mathrm{E}+00$ & $5.87 \mathrm{E}-02$ \\
\hline & En iyi & $1.78 \mathrm{E}-12$ & 8.63E-14 & $1.55 \mathrm{E}-12$ & $2.08 \mathrm{E}-13$ & $1.37 \mathrm{E}-12$ & $1.88 \mathrm{E}-12$ & $3.87 \mathrm{E}+02$ & $1.07 \mathrm{E}+00$ \\
\hline$F_{19}$ & Ortalama & 3.89E-12 & 7.39E-13 & $3.66 \mathrm{E}-12$ & $8.66 \mathrm{E}-12$ & $3.66 \mathrm{E}-12$ & $3.66 \mathrm{E}-12$ & $4.27 \mathrm{E}+02$ & $1.67 \mathrm{E}+00$ \\
\hline & S. S. & $2.46 \mathrm{E}-12$ & $4.70 \mathrm{E}-13$ & $5.17 \mathrm{E}-12$ & $7.90 \mathrm{E}-12$ & $5.17 \mathrm{E}-12$ & $5.27 \mathrm{E}-12$ & $4.08 \mathrm{E}+01$ & 4.35E-01 \\
\hline & En iyi & $9.11 \mathrm{E}-05$ & $1.98 \mathrm{E}-05$ & 1.74E-05 & $1.79 \mathrm{E}-05$ & $2.67 \mathrm{E}-05$ & $2.05 \mathrm{E}-05$ & $4.31 \mathrm{E}+01$ & $6.01 E+00$ \\
\hline$F_{20}$ & Ortalama & $1.30 \mathrm{E}-04$ & $8.43 \mathrm{E}-05$ & 3.59E-05 & 4.72E-05 & $9.50 \mathrm{E}-05$ & $5.90 \mathrm{E}-05$ & $4.69 \mathrm{E}+01$ & $6.34 \mathrm{E}+00$ \\
\hline & S. S. & $3.00 \mathrm{E}-05$ & $4.69 \mathrm{E}-05$ & 1.73E-05 & $2.07 \mathrm{E}-05$ & 7.19E-05 & $2.95 \mathrm{E}-05$ & $3.72 \mathrm{E}+00$ & $2.72 \mathrm{E}-01$ \\
\hline & En iyi & $1.78 \mathrm{E}-01$ & $1.28 \mathrm{E}-01$ & 1.26E-01 & $1.78 \mathrm{E}-01$ & $1.27 \mathrm{E}-01$ & $1.27 \mathrm{E}-01$ & 4.99E-01 & 4.29E-01 \\
\hline$F_{21}$ & Ortalama & $1.94 \mathrm{E}-01$ & $1.61 \mathrm{E}-01$ & 1.44E-01 & $1.94 \mathrm{E}-01$ & $1.54 \mathrm{E}-01$ & $1.44 \mathrm{E}-01$ & $5.90 \mathrm{E}-01$ & 4.43E-01 \\
\hline & S. S. & 2.33E-02 & $2.36 \mathrm{E}-02$ & $2.44 \mathrm{E}-02$ & 2.33E-02 & $2.11 \mathrm{E}-02$ & $2.39 \mathrm{E}-02$ & $1.70 \mathrm{E}-01$ & 2.97E-02 \\
\hline & En iyi & $-7.32 \mathrm{E}+01$ & $-7.38 E+01$ & $-7.21 \mathrm{E}+01$ & $-6.19 E+01$ & $-7.21 E+01$ & $-7.55 E+01$ & $-5.25 \mathrm{E}+01$ & $-7.22 \mathrm{E}+01$ \\
\hline$F_{22}$ & Ortalama & $-7.13 E+01$ & $-7.32 \mathrm{E}+01$ & $-6.96 E+01$ & $-6.11 E+01$ & $-6.96 E+01$ & $-7.08 E+01$ & $-5.14 \mathrm{E}+01$ & $-7.12 \mathrm{E}+01$ \\
\hline & S. S. & $1.40 \mathrm{E}+00$ & 7.99E-01 & $1.86 \mathrm{E}+00$ & $1.06 \mathrm{E}+00$ & $2.36 \mathrm{E}+00$ & $3.29 \mathrm{E}+00$ & 8.11E-01 & $9.46 \mathrm{E}-01$ \\
\hline & En iyi & $3.27 \mathrm{E}-17$ & 1.14E-18 & $6.09 \mathrm{E}-18$ & $3.30 \mathrm{E}-17$ & $9.12 \mathrm{E}-18$ & $5.76 \mathrm{E}-18$ & $8.18 \mathrm{E}-05$ & $1.03 \mathrm{E}-10$ \\
\hline$F_{23}$ & Ortalama & $1.68 \mathrm{E}-16$ & $2.34 \mathrm{E}-16$ & $2.81 \mathrm{E}-17$ & $3.84 \mathrm{E}-17$ & $2.59 \mathrm{E}-17$ & 1.75E-17 & $4.86 \mathrm{E}-02$ & $4.10 \mathrm{E}-10$ \\
\hline & S. S. & $3.14 \mathrm{E}-16$ & $1.09 \mathrm{E}-16$ & $1.64 \mathrm{E}-17$ & $3.80 \mathrm{E}-18$ & $1.74 \mathrm{E}-17$ & $9.13 \mathrm{E}-18$ & $5.60 \mathrm{E}-02$ & $3.95 \mathrm{E}-10$ \\
\hline & En iyi & $9.61 \mathrm{E}-01$ & $6.67 \mathrm{E}-01$ & $6.68 \mathrm{E}-01$ & $6.66 \mathrm{E}-01$ & $6.82 \mathrm{E}-01$ & $6.78 \mathrm{E}-01$ & $3.02 E+05$ & $7.46 \mathrm{E}+01$ \\
\hline$F_{24}$ & Ortalama & $3.03 E+00$ & $2.05 \mathrm{E}+00$ & $2.05 E+00$ & $7.20 \mathrm{E}-01$ & $1.83 \mathrm{E}+00$ & 1.13E-01 & $4.76 \mathrm{E}+05$ & $8.45 E+01$ \\
\hline & S. S. & $1.81 \mathrm{E}+00$ & $1.72 \mathrm{E}+00$ & 9.95E-01 & 7.56E-02 & $1.41 \mathrm{E}+00$ & $3.35 \mathrm{E}-01$ & $1.44 \mathrm{E}+05$ & $7.84 \mathrm{E}+00$ \\
\hline
\end{tabular}



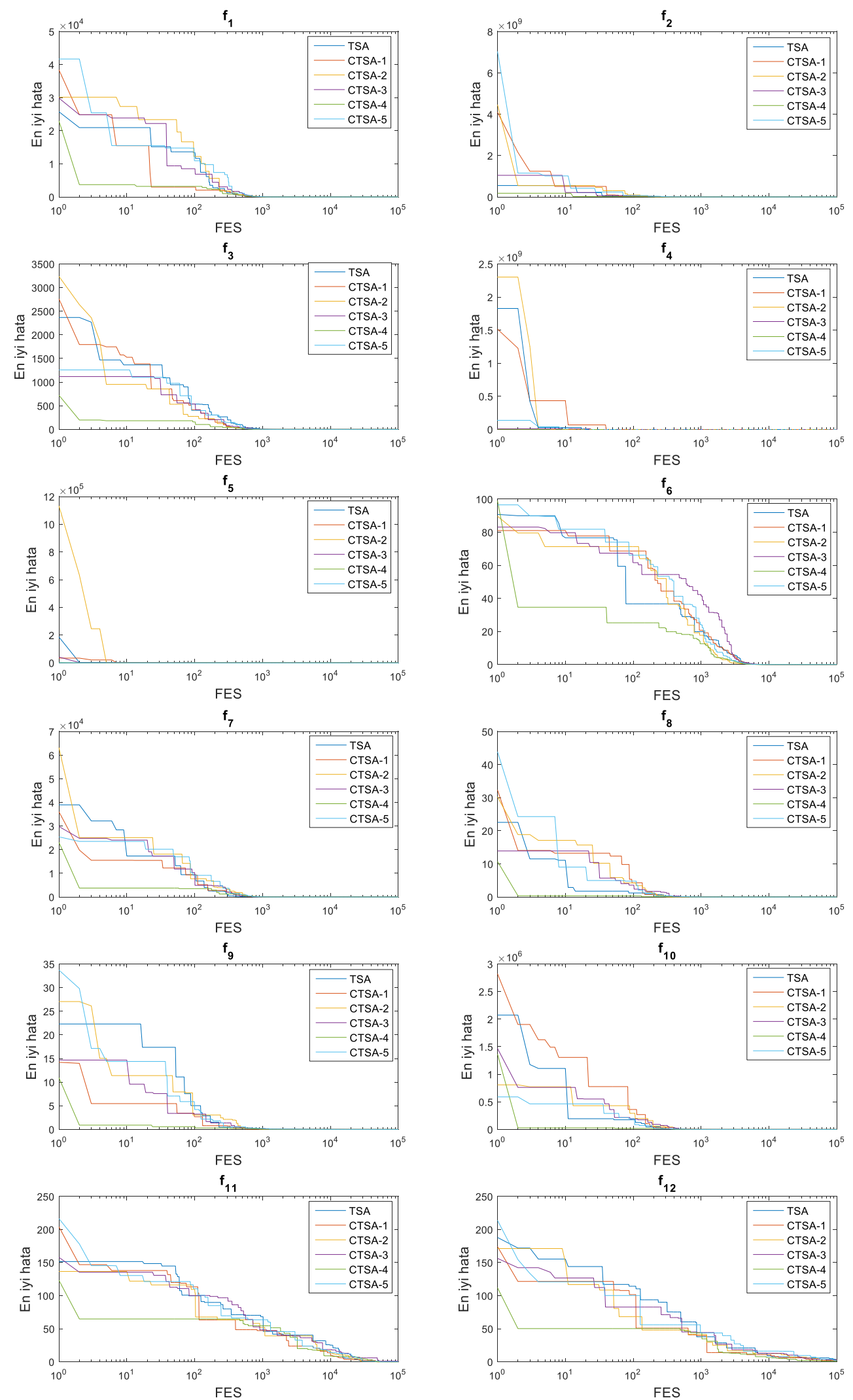

Şekil 6. $F_{1}-F_{12}$ fonksiyonlarına ait yakınsama eğrileri 

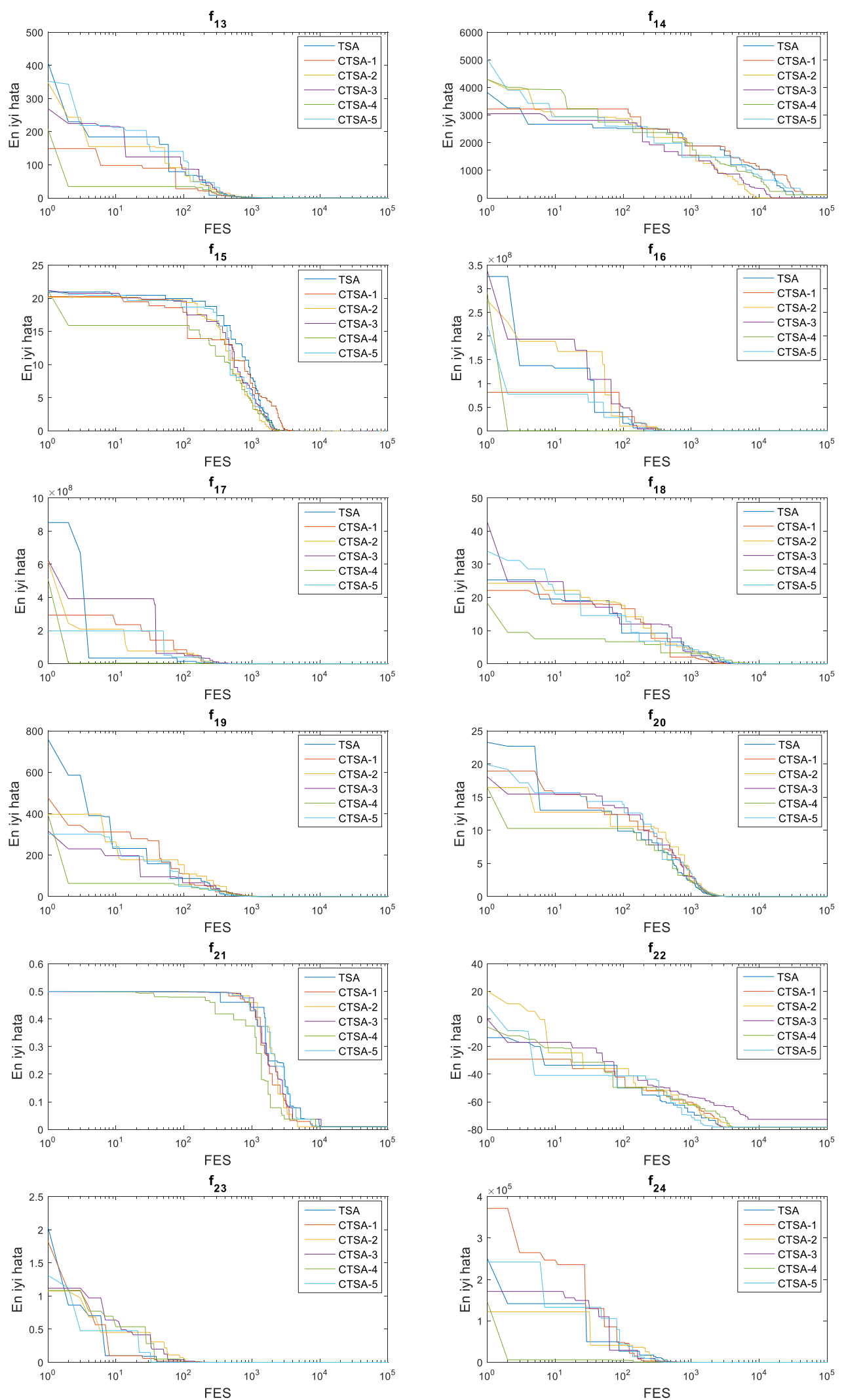

Şekil 7. $F_{13}-F_{24}$ fonksiyonlarına ait yakınsama eğrileri

\section{Kaynakça}

[1] Kennedy, J., Eberhart, R. 1995. Particle Swarm Optimization. IEEE International Conference on Neural Networks, 27 November-1 December 1995, Perth, 1942-1948.

[2] Dorigo, M., Caro, G. D. 1999. The Ant Colony Optimization Meta-Heuristic. ss11-32. Corne, D., Dorigo, M., Glover, F., ed. 1999. New Ideas in Optimization, McGraw-Hill, New York, 493s.

[3] Karaboga, D., Basturk, B. 2007. A Powerful and Efficient Algorithm for Numerical Function Pptimization: Artificial Bee Colony (ABC) 
Algorithm. Journal of Global Optimization, 39(3), 459-471.

[4] Yang, X. S. 2012. Flower Pollination Algorithm for Global Optimization. Lecture Notes in Computer Science, 7445, 240-249.

[5] Yazdani, M., Jolai, F. 2016. Lion Optimization Algoritgm (LOA): A Nature-Inspired Metaheuristic Algorithm. Journal of Computational Design and Engineering, 3(1), 24-36.

[6] Gandomi, A. H., Yang, X. S., Talatahari, S., Alavi, A. H. 2013. Firefly Algorithm with Chaos. Communications in Nonlinear Science and Numerical Simulation, 18(1), 89-98.

[7] Han, X., Chang, X. 2012. A Chaotic Digital Secure Communication Based on A Modified Gravitational Search Algorithm Filter. Information Sciences, 208, 14-27.

[8] Wang, G. G., Guo, L., Gandomi, A. H., Hao, G. S., Wang, H. 2014. Chaotic Krill Herd Algorithm. Information Sciences, 274, 17-34.

[9] Alataş, B. 2010. Chaotic Harmony Search Algorithms. Applied Mathematics and Computation 2 216(9), 2687-2699.

[10] Askarzadeh, A., Coelho, L. S. 2014. A Backtracking Search Algorithm Combined with Burger's Chaotic Map for Parameter Estimation of PEMFC Electrochemical Model. International Journal of Hydrogen Energy, 39(21), 1116511174.

[11] Kaur, G., Arora, S. 2018. Chaotic Whale Optimization Algorithm. Journal of Computational Design and Engineering, 5(3), 275-284.

[12] Kohli, M., Arora, S. 2018. Chaotic Grey Wolf Optimization Algorithm for Constrained Optimization Problems. Journal of Computational Design and Engineering, 5(4), 458-472.

[13] Yüzgeç, U., Eser, M. 2018. Chaotic based Differential Evolution Algorithm for Optimization of Baker's Yeast Drying Process. Egyptian Informatics Journal, 19(3),151-163.

[14] Feng, J., Zhang, J., Zhu, X., Lian, W. 2017. A Novel Chaos Optimization Algorithm. Multimedia Tools and Applications, 76(16),17405-17436.

[15] Saremi, S., Mirjalili, S., Lewis, A. 2014. Biogeography-based Optimisation with Chaos. Neural Computing and Applications, 25(5), 1077-1097.
[16] Kiran, M. S. 2015. TSA: Tree-Seed Algorithm for Continuous Optimization. Expert Systems with Applications, 42, 6686-6698.

[17] Cinar, A. C., Kiran M. S. 2018. Ağaç-Tohum Algoritmasının CUDA Destekli Grafik İşlem Birimi Üzerinde Paralel Uygulaması. Journal of Faculty of Engineering and Architecture of Gazi University, 33(4), 1397-1409.

[18] Babalık, A., Çınar, A. C., Kıran, M. S. 2018. A Modification of Tree-Seed Algorithm using Deb's Rules for Constrained Optimization. Applied Soft Computing, 63, 289-305.

[19] Cınar, A. C., Kıran, M. S. 2018. Similarity and Logic Gate-Based Tree-Seed Algorithms for Binary Optimization. Computers \& Industrial Engineering, 115, 631-646.

[20] Hilborn, R. C. 2004. Chaos and Nonlinear Dynamics: An Introduction for Scientists and Engineers. 2nd, Oxford University Press, New York, 672s.

[21] Mondragon, R. J., Pitts, J. M., Arrowsmith, D. K. 2000. Chaotic Intermittency-Sawtooth Map Model of Aggregate Self-Similar Traffic Streams. IEEE Electronics Letters, 36(2), 184-186.

[22] Li, Y., Deng, S., Xiao, D. 2011. A novel Hash Algorithm Construction Based on Chaotic Neural Network. Neural Computing and Application, 20, 133-141.

[23] Chirikov, B. V. 1979. A Universal Instability of Many-Dimensional Oscillator Systems. Physics Reports, 52(5), 263-379.

[24] Zaslavskii, G. M. 1978. The Simplest Case of A Strange Attractor. Physics Letters A, 69(3), 145147.

[25] Karaboğa, D., Akay, B. 2009. A Comparative Study of Artificial Bee Colony Algorithm. Applied Mathematics and Computation, 214, 108-132.

[26] Boyer, D. O., Martfnez, C. H., Pedrajas, N. G. 2005. Crossover Operator for Evolutionary Algorithms Based on Population Features. Journal of Artificial Intelligence Research, 24, 1-48.

[27] Digalakis, J. G., Margaritis, K. G. 2002. An Experimental Study of Benchmarking Functions for Genetic Algorithms. International Journal of Computer Mathematics, 79(4), 403-416.

[28] Yao, X., Liu, Y., Lin, G. 1999. Evolutionary Programming Made Faster. IEEE Transactions on Evolutionary Computation, 3(2), 82-102. 